\title{
Four-dimensional unsubtraction with massive particles
}

\author{
Germán F.R. Sborlini, Félix Driencourt-Mangin and Germán Rodrigo \\ Instituto de Física Corpuscular, Universitat de València, \\ Consejo Superior de Investigaciones Cientificas, \\ Parc Cientific, Paterna, Valencia, E-46980 Spain \\ E-mail: german.sborlini@ific.uv.es, felix.dm@ific.uv.es, \\ german.rodrigo@csic.es
}

ABSTRACT: We extend the four-dimensional unsubtraction method, which is based on the loop-tree duality (LTD), to deal with processes involving heavy particles. The method allows to perform the summation over degenerate IR configurations directly at integrand level in such a way that NLO corrections can be implemented directly in four spacetime dimensions. We define a general momentum mapping between the real and virtual kinematics that accounts properly for the quasi-collinear configurations, and leads to an smooth massless limit. We illustrate the method first with a scalar toy example, and then analyse the case of the decay of a scalar or vector boson into a pair of massive quarks. The results presented in this paper are suitable for the application of the method to any multipartonic process.

KEYwORDS: NLO Computations

ARXIV EPRINT: 1608.01584 


\section{Contents}

1 Introduction 1

2 Loop-tree duality: concepts and notation 3

3 Massive scalar three-point function within LTD 5

4 Massive scalar decay rate in DREG $\quad 9$

5 Phase-space partition and real-virtual mapping with massive particles 11

$\begin{array}{lll}5.1 \text { General momentum mapping } & 15\end{array}$

6 Massive scalar decay rate from four-dimensional unsubstraction $\quad 16$

7 Unintregrated wave function and mass renormalisation for heavy quarks 18

8 UV renormalisation $\quad 20$

$9 \quad$ LTD four-dimensional unsubtraction for physical processes 23

10 Conclusions and outlook $\quad 24$

$\begin{array}{lr}\text { A Phase-space } & 26\end{array}$

$\begin{array}{ll}\text { B Unification of coordinates } & 27\end{array}$

$\begin{array}{ll}\text { C LTD amplitudes for } A^{*} \rightarrow q \bar{q}(g) & 28\end{array}$

\section{Introduction}

The development of new computational techniques to obtain more accurate theoretical predictions at colliders has been strongly pushed forward by the high precision experimental data obtained from the LHC. In the framework of perturbative quantum field theory (and perturbative QCD in particular), the presence of infrared (IR) and ultraviolet (UV) divergences constitutes the main difficulty that must be overcome to obtain physical results. Renormalisation has been proven to deal successfully with the UV structure of these theories [1], and the cancellation of IR singularities is guaranteed by general theorems $[2,3]$ for physical observables that sum over all the possible degenerate IR configurations. This requires taking into account both loop scattering amplitudes and real processes with the emission of additional external particles; the sum of all contributions leads to IR-safe observables. On the other hand, UV divergences are removed by suitable counter-terms, whose divergent structure depends only on the specific theory under consideration. 
In order to make these divergences manifest explicitly, the standard approach relies in the introduction of a convenient regularisation method. Nowadays, the standard choice in gauge theories is dimensional-regularisation (DREG) [4-7] because it preserves gauge invariance. Within DREG, the space-time is analytically continued from $d=4$ to $d=4-2 \epsilon$ dimensions; both UV and IR divergences appear as $\epsilon$-poles. Using $\epsilon$ as a regulator, it is possible to compute the loop and the phase-space integrals for the virtual and real-radiation amplitudes, respectively. Thus, the poles of the virtual corrections are cancelled with those present in the real-radiation contributions (due to soft/collinear configurations) and those included in the UV counter-terms. The usual procedure in this framework is to regularise each contribution separately, integrate the expressions and, finally, cancel the $\epsilon$ poles and take the limit $\epsilon \rightarrow 0$.

Besides the renormalisation and the regularisation method, the fact that real and virtual contributions have the same IR-divergent behaviour is the underlying basis of the subtraction methods [8-11]. The general idea of subtraction is the introduction of counterterms which mimic the local IR behaviour of the real components and that can easily be integrated analytically. In this way, the integrated form is combined with the virtual component whilst the unintegrated counter-term cancels the IR poles originated from the phase-space integration of the real-radiation part. The subtraction paradigm has evolved to different versions from the FKS-subtraction [8, 9], and dipole-subtraction [10, 11], to antenna-subtraction [12], $q_{T}$-subtraction $[14,15]$ and other recent variations [16-22]. The treatment of massive particles has also been considered specifically [23-27]. However, all these approaches treat separately real and virtual corrections, since the final-state phasespace of the different contributions involves different numbers of particles. The construction of IR counter-terms is inherent to the subtraction approach, and it constitutes the main restriction for an efficient application to multi-leg multi-loop processes.

With the purpose of obtaining a fully-local cancellation of IR singularities without building IR-counter-terms, we apply the loop-tree duality (LTD) theorem [28-31] to handle the virtual corrections. This theorem asserts that loop integrals are expressible as the sum of dual integrals, which are built starting from tree-level like objects and replacing the loop measure with an extended phase-space measure. The main advantage of LTD is that by introducing additional physical on-shell particles, dual integrals and real-radiation contributions exhibit a similar structure. Moreover, the loop threshold and IR singularities are always restricted to a compact region of the loop three-momentum space [32, 33]. These two properties of LTD allow a natural integrand-level combination of virtual and real corrections. The method has been recently developed for processes involving massless particles in refs. [36-40]. The key point in this framework is the establishment of a momentum mapping to generate the real-radiation kinematics from the Born and the loop momenta. In this way, we guarantee not only a simultaneous cancellation of IR singularities without the necessity of introducing IR subtractions but also a fully-local four-dimensional implementation.

Before describing the content of this article, we would like to highlight that many other attempts have been previously developed to obtain four-dimensional representations of higher-order corrections to physical observables. In refs. [41-44] it was proposed to cancel 
the singularities through the application of a momentum smearing to relate real and virtual kinematics. In this way, both real and virtual terms could be combined at the integrand level, thus achieving a local cancellation of singularities. Other alternative methods consist in rewriting the standard IR/UV subtraction counter-terms in a local form, as discussed in refs. [45, 46], or modifying the structure of the propagators and the Feynman rules [4749] to regularise the singularities at integrand level. LTD has also been used recently to deal with integral representations of virtual and real subtraction terms [13], including the description of initial-state singularities that are grouped together and then are integrated numerically.

The main purpose of this article is to extend the LTD four-dimensional unsubtraction method presented in refs. [36-40] to deal with massive particles. From the kinematical point of view, the mass of the particles slightly modifies the momentum mapping used to perform the real-virtual combination and this changes the IR-divergent structure. The major difference comes from the treatment of the self-energy corrections, since they must fulfill non-trivial constraints both in the IR and UV regions. Moreover, quasi-collinear configurations are mapped in such a way that logarithmic contributions are cancelled at the integrand level and the massless limit is smooth. In any case, the dual representations involve dealing with higher-order poles in LTD [30, 31]. We restrict the discussion of the treatment of massive partons to the final state because the validity of the QCD factorization theorems requires that the partons that initiate the hard-scattering subprocess in hadronic collisions have to be massless [23]. The treatment of initial state radiation with massless partons, the last necessary ingredient to have a full description of hadronic collisions in LTD, is deferred for a forthcoming publication.

The outline of this article is the following. In section 2 we set the notation and briefly recall some results related with the LTD theorem. After that, in section 3, we apply LTD to study the one-loop scalar three-point function with massive particles. In section 4, we introduce an scalar toy example and compute NLO corrections through the application of the conventional DREG approach. After describing the real emission phasespace partition and introducing a proper momentum mapping in section 5 , we deal with the NLO corrections of the scalar toy model within the LTD approach in section 6 . We use LTD and the momentum mapping to perform the real-virtual combination at integrand level and define purely four-dimensional integrable expressions. In section 7, we present integral representations for the wave function and mass renormalisation factors for heavy quarks in the on-shell renormalisation scheme. Renormalisation is then discussed in section 8 . Afterwards, we proceed to implement this technique to deal with physical theories. In particular, we compute the NLO QCD corrections to the decay rate $A^{*} \rightarrow q \bar{q}(g)$, with massive quarks and $A=\{\phi, \gamma, Z\}$. The results are presented in section 9 , emphasising the four-dimensional nature of the implementation. Finally, in section 10, the conclusions are exposed and we discuss future implications of this work.

\section{Loop-tree duality: concepts and notation}

In this section, we summarise the key concepts of the LTD theorem at one-loop. So, let's consider a generic one-loop scalar integral for an $N$-particle process, as depicted in figure 1 . 


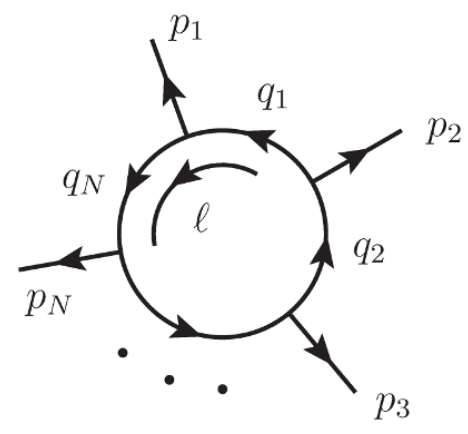

Figure 1. One-loop topology with $N$ external legs and the corresponding momenta configuration. The external momenta are considered outgoing and the internal momentum flows counter-clockwise.

If the external momenta are labeled as $p_{i}$ with $i \in\{1,2, \ldots N\}$, then the internal virtual momenta are given by

$$
q_{i}=\ell+k_{i}, \quad k_{i}=p_{1}+\ldots+p_{i},
$$

with $\ell$ the loop internal momentum and $k_{N}=0$ due to momentum conservation. The corresponding expression for the scalar integral is

$$
L^{(1)}\left(p_{1}, \ldots, p_{N}\right)=\int_{\ell} \prod_{i=1}^{N} G_{\mathrm{F}}\left(q_{i}\right),
$$

where

$$
G_{\mathrm{F}}\left(q_{i}\right)=\frac{1}{q_{i}^{2}-m_{i}^{2}+\imath 0},
$$

is the scalar Feynman propagator associated to a virtual particle with mass $m_{i}$ and fourmomentum $q_{i, \mu}=\left(q_{i, 0}, \mathbf{q}_{i}\right)$ ( $q_{i, 0}$ is the energy and $\mathbf{q}_{i}$ are the spatial components). We recall that the $+\imath 0$ prescription is introduced to separate, in the imaginary axis, the solutions arising from the on-shell condition, i.e. $G_{\mathrm{F}}\left(q_{i}\right)^{-1}=0$. In particular, this translates into two solutions with positive and negative imaginary components, respectively. On the other hand,

$$
\int_{\ell}=-\imath \mu^{4-d} \int \frac{d^{d} \ell}{(2 \pi)^{d}}
$$

denotes the standard one-loop integration measure in $d$-dimensions.

According to the LTD theorem, any loop contribution to scattering amplitudes in any relativistic, local and unitary quantum field theory can be computed through dual integrals, which are built from single cuts of the virtual diagrams at one-loop [28]. In other words, there exists a formal connection among loop and phase-space integrals. The cut condition is implemented by restricting the integration measure through the introduction of

$$
\tilde{\delta}\left(q_{i}\right) \equiv 2 \pi \imath \theta\left(q_{i, 0}\right) \delta\left(q_{i}^{2}-m_{i}^{2}\right),
$$

which forces to integrate in the positive energy mode $\left(q_{i, 0}>0\right)$ of the corresponding onshell hyperboloid (i.e. $q_{i}^{2}=m_{i}^{2}$ ). It is worth appreciating that, in the massless limit, all 
the hyperboloids associated with the on-shell condition $G_{\mathrm{F}}\left(q_{i}\right)^{-1}=0$ degenerate into lightcones. Considering the one-loop scalar integral, the LTD theorem establishes that its dual representation is given by

$$
L^{(1)}\left(p_{1}, \ldots, p_{N}\right)=-\sum_{i=1}^{N} \int_{\ell} \tilde{\delta}\left(q_{i}\right) \prod_{j \neq i} G_{\mathrm{D}}\left(q_{i} ; q_{j}\right),
$$

i.e. the sum of $N$ dual integrals, each one associated with one of the possible single-cuts. In eq. (2.6), the dual propagators are

$$
G_{\mathrm{D}}\left(q_{i} ; q_{j}\right)=\frac{1}{q_{j}^{2}-m_{j}^{2}-\imath 0 \eta \cdot k_{j i}},
$$

with $i, j \in\{1,2, \ldots N\}, k_{j i}=q_{j}-q_{i}$ and $\eta$ an arbitrary future-like or light-like vector, $\eta^{2} \geq 0$, with positive definite energy $\eta_{0}>0 .{ }^{1}$ Since $\eta$ is arbitrary, we can chose $\eta^{\mu}=(1, \mathbf{0})$ to simplify the computations.

Assuming that there are only single powers of the Feynman propagators, the dual representation in eq. (2.6) is straightforwardly valid for loop scattering amplitudes. The single-cuts do not affect numerators, therefore, the dual representation of scattering amplitudes is obtained by simply adding all possible dual single-cuts of the original loop diagram, and replacing the uncut Feynman propagators by dual propagators. If there are higher-powers of the propagators, however, we should apply the extended version of the LTD theorem [31] by using the Cauchy's residue theorem with the well-known formula for poles of order $n$, i.e.

$$
\operatorname{Res}\left(\mathcal{A}, q_{i, 0}^{(+)}\right)=\left.\frac{1}{(n-1) !} \frac{\partial^{n-1}}{\partial^{n-1} q_{i, 0}}\left(\mathcal{A}\left(q_{i, 0}\right)\left(q_{i, 0}-q_{i, 0}^{(+)}\right)^{n}\right)\right|_{q_{i, 0}=q_{i, 0}^{(+)}},
$$

where $q_{i, 0}^{(+)}=\sqrt{\mathbf{q}_{i}^{2}+m_{i}^{2}-\imath 0}$ is the positive energy solution of the corresponding on-shell dispersion relation. In that case, the explicit form of the scattering amplitude is relevant because the numerator is affected by the derivative.

\section{Massive scalar three-point function within LTD}

We present in this section the first analytical application of LTD with massive particles. In particular, we consider the scalar three-point function with one massless internal state and the remaining internal and outgoing particles with mass equal to $M$. The final-state on-shell momenta are labeled as $p_{1}$ and $p_{2}$, with $p_{1}^{2}=M^{2}=p_{2}^{2}$, and the incoming one is $p_{3}=p_{1}+p_{2} \equiv p_{12}$, by momentum conservation, with virtuality $p_{3}^{2}=s_{12}$. We consider $s_{12}>0$, i.e. we work in the time-like (TL) kinematical region. The internal momenta are $q_{1}=\ell+p_{1}, q_{2}=\ell+p_{12}$ and $q_{3}=\ell$, where $\ell$ is the loop momentum (see figure 3 ). When the internal lines are set on-shell, the momentum $q_{1}$ is massless, whilst the other two are

\footnotetext{
${ }^{1}$ The importance of the modified prescription is deeply explored in ref. [28], where the authors emphasise its equivalence with the Feynman-Tree theorem (FTT) [50, 51].
} 
massive and fulfill $q_{2}^{2}=M^{2}=q_{3}^{2}$. This is the master scalar configuration for the calculation of the QCD corrections to the physical case $A^{*} \rightarrow q \bar{q}(g)$ with massive quarks that will be studied in section 9 . We define

$$
m=\frac{2 M}{\sqrt{s_{12}}}, \quad \beta=\sqrt{1-m^{2}},
$$

as the normalised mass and velocity, respectively. The well-know result of this massive scalar three-point function is given by $[52,53]$

$$
\begin{aligned}
L_{m>0}^{(1)}\left(p_{1}, p_{2},-p_{3}\right)=\int_{\ell_{i=1}^{3}}^{3} G_{\mathrm{F}}\left(q_{i}\right)= & -\frac{c_{\Gamma}}{s_{12} \beta}\left[\operatorname { l o g } ( X _ { S } ) \left(-\frac{1}{\epsilon}-\frac{\log \left(X_{S}\right)}{2}+2 \log \left(1-X_{S}^{2}\right)\right.\right. \\
& \left.\left.+\log \left(\frac{m^{2}}{4}\right)\right)+\operatorname{Li}_{2}\left(X_{S}^{2}\right)+2 \operatorname{Li}_{2}\left(1-X_{S}\right)-\frac{\pi^{2}}{6}\right]+\mathcal{O}(\epsilon),
\end{aligned}
$$

with

$$
X_{S}=-x_{S}-\imath 0 \operatorname{Sgn}\left(s_{12}\right), \quad x_{S}=\frac{1-\beta}{1+\beta},
$$

and $c_{\Gamma}$ the one-loop volume factor

$$
c_{\Gamma}=\frac{\Gamma(1+\epsilon) \Gamma^{2}(1-\epsilon)}{(4 \pi)^{2-\epsilon} \Gamma(1-2 \epsilon)} .
$$

Applying LTD, the dual representation of the scalar integral in eq. (3.2) consists of three contributions

$$
L_{m>0}^{(1)}\left(p_{1}, p_{2},-p_{3}\right)=\sum_{i=1}^{3} I_{i},
$$

with

$$
\begin{aligned}
& I_{1}=-\int_{\ell} \frac{\tilde{\delta}\left(q_{1}\right)}{\left(2 q_{1} \cdot p_{2}-\imath 0\right)\left(-2 q_{1} \cdot p_{1}+\imath 0\right)}, \\
& I_{2}=-\int_{\ell} \frac{\tilde{\delta}\left(q_{2}\right)}{\left(2 M^{2}-2 q_{2} \cdot p_{2}+\imath 0\right)\left(s_{12}-2 q_{2} \cdot p_{12}+\imath 0\right)}, \\
& I_{3}=-\int_{\ell} \frac{\tilde{\delta}\left(q_{3}\right)}{\left(2 M^{2}+2 q_{3} \cdot p_{1}-\imath 0\right)\left(s_{12}+2 q_{3} \cdot p_{12}-\imath 0\right)} .
\end{aligned}
$$

The corresponding on-shell hyperboloids are shown in figure 2 (left). Due to the rotational symmetry, it is enough to show the $\left(\ell_{0}, \ell_{z}\right)$ plane. As discussed in ref. [32], the intersection of on-shell hyperboloids is associated with multiple internal states becoming simultaneously on-shell. In this case, the forward on-shell hyperboloids of $G_{\mathrm{F}}\left(q_{1}\right)$ and $G_{\mathrm{F}}\left(q_{2}\right)$, and the backward one of $G_{\mathrm{F}}\left(q_{3}\right)$ intersect in a single point: this leads to a soft singularity. The other intersection takes place between the forward on-shell hyperboloid of $G_{\mathrm{F}}\left(q_{2}\right)$ with the backward of $G_{\mathrm{F}}\left(q_{3}\right)$, which manifests as a threshold singularity in $I_{2}$. Notice that there 

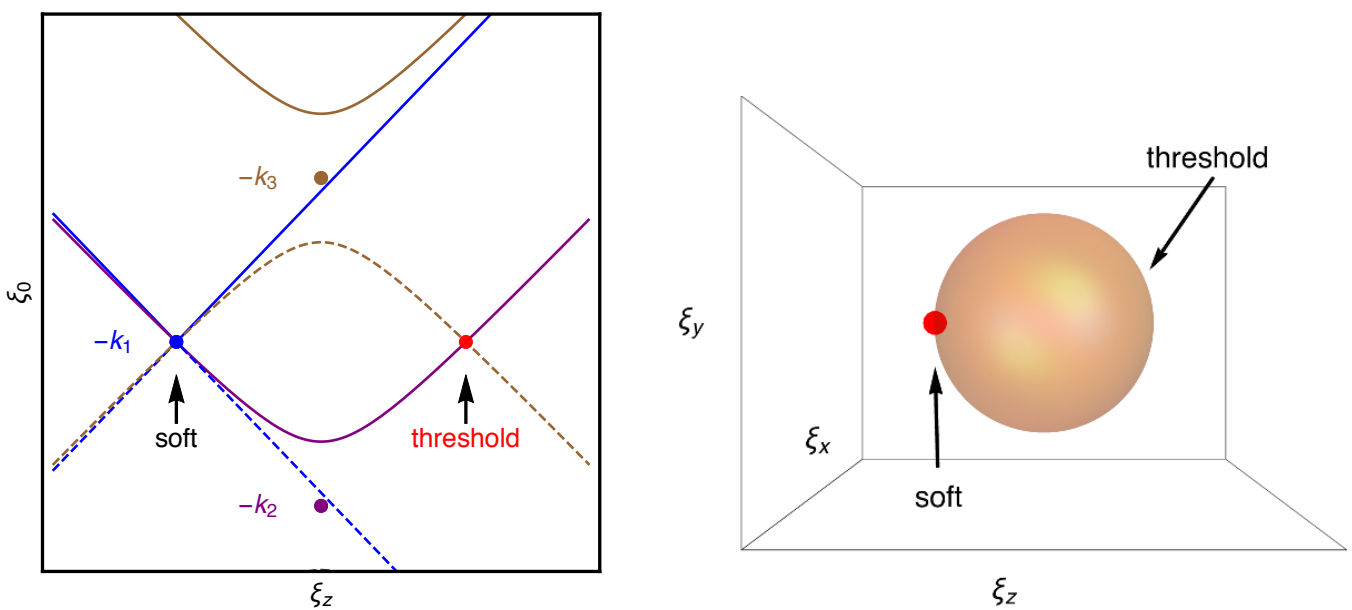

Figure 2. On-shell hyperboloids of the massive three-point function in the loop coordinates $\ell^{\mu}=$ $\sqrt{s_{12}} / 2\left(\xi_{0}, \xi_{x}, \xi_{y}, \xi_{z}\right)$ in two dimensions (left plot); forward and backward on-shell hyperboloids are represented by solid and dashed lines, respectively. The intersection of on-shell hyperboloids leads to soft and threshold singularities in the loop three-momentum space (right plot), collinear singularities are regulated by the mass.

are not collinear singularities, because the mass prevent that the on-shell hyperboloids degenerate into light-cones. In that situation, there would be extended forward-backward intersections in the $\left(\ell_{0}, \ell_{z}\right)$ plane leading to collinear poles, as described in the massless case studied in refs. [36, 39].

Now, we shall explicitly compute these dual integrals. We choose first a proper reference frame to simplify the analytic expressions. Hence, we work in the centre-of-mass frame of $p_{1}$ and $p_{2}$, and parametrise the involved momenta as

$$
p_{1}^{\mu}=\frac{\sqrt{s_{12}}}{2}\left(1, \mathbf{0}_{\perp}, \beta\right), \quad p_{2}^{\mu}=\frac{\sqrt{s_{12}}}{2}\left(1, \mathbf{0}_{\perp},-\beta\right) .
$$

In order to describe the internal on-shell momenta, we must take into account that $q_{1}$ corresponds to a massless state $\left(q_{1}^{2}=0\right)$, whilst $q_{2}$ and $q_{3}$ are associated with massive virtual particles $\left(q_{2}^{2}=M^{2}=q_{3}^{2}\right)$. For this reason, we write

$$
\begin{aligned}
& q_{1}^{\mu}=\frac{\sqrt{s_{12}}}{2} \xi_{1,0}\left(1,2 \sqrt{v_{1}\left(1-v_{1}\right)} \mathbf{e}_{1, \perp}, 1-2 v_{1}\right), \\
& q_{i}^{\mu}=\frac{\sqrt{s_{12}}}{2}\left(\xi_{i, 0}, 2 \xi_{i} \sqrt{v_{i}\left(1-v_{i}\right)} \mathbf{e}_{i, \perp}, \xi_{i}\left(1-2 v_{i}\right)\right), \quad \xi_{i, 0}=\sqrt{m^{2}+\xi_{i}^{2}}, \quad i=\{2,3\},
\end{aligned}
$$

where $\xi_{1,0}, \xi_{2}, \xi_{3} \in[0, \infty)$ and $v_{i} \in[0,1]$ are the integration variables describing the modulus of the three-momentum and polar angle of the loop momenta, respectively. With these variables, the LTD transforms the loop integration measure into

$$
\int_{\ell} \tilde{\delta}\left(q_{i}\right)=s_{12} \int_{0}^{\infty} \frac{\xi_{i}^{2}}{\xi_{i, 0}} d\left[\xi_{i}\right] \int_{0}^{1} d\left[v_{i}\right]
$$


with

$$
d\left[\xi_{i}\right]=\frac{(4 \pi)^{\epsilon-2}}{\Gamma(1-\epsilon)}\left(\frac{s_{12}}{\mu^{2}}\right)^{-\epsilon} \xi_{i}^{-2 \epsilon} d \xi_{i}, \quad d\left[v_{i}\right]=\left(v_{i}\left(1-v_{i}\right)\right)^{-\epsilon} d v_{i},
$$

for the massive case $(i=2,3)$, whilst it reduces to

$$
\int_{\ell} \tilde{\delta}\left(q_{1}\right)=s_{12} \int_{0}^{\infty} \xi_{1,0} d\left[\xi_{1,0}\right] \int_{0}^{1} d\left[v_{1}\right]
$$

for a massless state $(i=1)$. Notice that $\xi_{1,0}=\xi_{1}$ since $q_{1}$ is massless. The integration of the loop momentum in the transverse plane, which is described by the unit vectors $\mathbf{e}_{i, \perp}$ is trivial. The scalar products of internal with external momenta are given by

$$
\begin{aligned}
& 4 q_{i} \cdot p_{1} / s_{12}=\xi_{i, 0}-\beta \xi_{i}\left(1-2 v_{i}\right), \\
& 4 q_{i} \cdot p_{2} / s_{12}=\xi_{i, 0}+\beta \xi_{i}\left(1-2 v_{i}\right),
\end{aligned}
$$

which reduce to $2 q_{1} \cdot p_{1} / s_{12}=\xi_{1,0} v_{1}$ and $2 q_{1} \cdot p_{2} / s_{12}=\xi_{1,0}\left(1-v_{1}\right)$ for a massless on-shell state. Using these variables, the dual integrals in eq. (3.6) are rewritten as

$$
\begin{aligned}
I_{1} & =\frac{4}{s_{12}} \int \frac{\xi_{1,0}^{-1} d\left[\xi_{1,0}\right] d\left[v_{1}\right]}{1-\left(1-2 v_{1}\right)^{2} \beta^{2}}, \\
I_{2} & =\frac{2}{s_{12}} \int \frac{\xi_{2}^{2} d\left[\xi_{2}\right] d\left[v_{2}\right]}{\xi_{2,0}\left(1-\xi_{2,0}+\imath 0\right)\left(\xi_{2,0}+\beta \xi_{2}\left(1-2 v_{2}\right)-m^{2}\right)}, \\
I_{3} & =-\frac{2}{s_{12}} \int \frac{\xi_{3}^{2} d\left[\xi_{3}\right] d\left[v_{3}\right]}{\xi_{3,0}\left(1+\xi_{3,0}\right)\left(\xi_{3,0}-\beta \xi_{3}\left(1-2 v_{3}\right)+m^{2}\right)} .
\end{aligned}
$$

Notice that $I_{2}$ contains a threshold singularity at $\sqrt{m^{2}+\xi_{2}^{2}}=1$, i.e. $\xi_{2}=\beta \leq 1$. These integrals can be calculated analytically to all orders in $\epsilon$ in the massless limit [36]. In this limit, they read

$$
\begin{aligned}
& I_{1}(m=0)=0 \\
& I_{2}(m=0)=\widetilde{c}_{\Gamma} \frac{\mu^{2 \epsilon}}{\epsilon^{2}} s_{12}^{-1-\epsilon} e^{\imath 2 \pi \epsilon} \\
& I_{3}(m=0)=\widetilde{c}_{\Gamma} \frac{\mu^{2 \epsilon}}{\epsilon^{2}} s_{12}^{-1-\epsilon}
\end{aligned}
$$

with $\widetilde{c}_{\Gamma}=c_{\Gamma} / \cos (\pi \epsilon)$ the phase-space volume factor. As expected, the sum of the three dual integrals in eq. (3.14) agrees with the well-known massless scalar three-point function.

The massive case is a bit more cumbersome because of the dependence on $m$. Again, $I_{1}$ vanishes because the energy integral factorises and it lacks of any characteristic scale. Actually, $I_{1}$ is singular both in the IR and UV. However, the sum of the three dual integrals and the equivalent original Feynman integral contain only soft divergences. The other two dual integrals can be integrated in the angular variable analytically, thus keeping the exact $\epsilon$-dependence. This leads to

$$
\begin{aligned}
& I_{2}=\frac{2 \Gamma^{2}(1-\epsilon)}{s_{12} \Gamma(2-2 \epsilon)} \int d\left[\xi_{2}\right] \frac{\xi_{22}^{2} F_{1}\left(1,1-\epsilon ; 2-2 \epsilon ; 2 \beta \xi_{2}\left(\xi_{2,0}+\beta \xi_{2}-m^{2}\right)^{-1}\right)}{\xi_{2,0}\left(1-\xi_{2,0}+\imath 0\right)\left(\xi_{2,0}+\beta \xi_{2}-m^{2}\right)} \\
& I_{3}=-\frac{2 \Gamma^{2}(1-\epsilon)}{s_{12} \Gamma(2-2 \epsilon)} \int d\left[\xi_{3}\right] \frac{\xi_{32}^{2} F_{1}\left(1,1-\epsilon ; 2-2 \epsilon ;-2 \beta \xi_{3}\left(\xi_{3,0}-\beta \xi_{3}+m^{2}\right)^{-1}\right)}{\xi_{3,0}\left(1+\xi_{3,0}\right)\left(\xi_{3,0}-\beta \xi_{3}+m^{2}\right)}
\end{aligned}
$$


However, an expansion in $\epsilon$ is necessary to integrate in the modulus of the loop threemomentum, which leads to a final result that includes corrections up to $\mathcal{O}\left(\epsilon^{0}\right)$. Besides that, the two dual integrals eq. (3.15) are singular only in the UV. Therefore, we introduce the following expansion

$$
I_{i}=\int \frac{d\left[\xi_{i}\right]}{\xi_{i, 0}} g_{i}\left(\xi_{i}\right)=\int \frac{d\left[\xi_{i}\right]}{\xi_{i, 0}} g_{\mathrm{UV}}+\left.\int \frac{d\left[\xi_{i}\right]}{\xi_{i, 0}}\left(g_{i}\left(\xi_{i}\right)-g_{\mathrm{UV}}\right)\right|_{\epsilon=0},
$$

with $g_{\mathrm{UV}}=\lim _{\xi_{i} \rightarrow \infty} g_{i}\left(\xi_{i}\right)$. The first term in the r.h.s. of eq. (3.16) gives the same result for both dual integrals, i.e.

$$
\int \frac{d\left[\xi_{i}\right]}{\xi_{i, 0}} g_{\mathrm{UV}}=-\frac{c_{\Gamma}}{s_{12}} \frac{x_{s}^{-\epsilon}\left(1+x_{s}\right)^{1+2 \epsilon} \Gamma(1-2 \epsilon)}{2 \epsilon(1-2 \epsilon) \Gamma^{2}(1-\epsilon)}{ }_{2} F_{1}\left(1,1-\epsilon ; 2-2 \epsilon, 1-x_{s}\right), \quad i=\{2,3\} .
$$

For the second term, which is regular in the UV, we perform the change of variable

$$
\xi_{i}=\frac{m}{2}\left(z-\frac{1}{z}\right)
$$

with $z \in[1, \infty)$. The total result for the real part of the two dual integrals, up to $\mathcal{O}(\epsilon)$, reads

$$
\begin{aligned}
\operatorname{Re}\left(I_{2}\right) & =\frac{c_{\Gamma}}{2 \beta s_{12}}\left(\frac{s_{12}}{\mu^{2}}\right)^{-\epsilon}\left[\log \left(x_{S}\right)\left(\frac{1}{\epsilon}-\frac{1}{2} \log \left(x_{S}\right)-2 \log (\beta)\right)-2 \operatorname{Li}_{2}\left(x_{S}\right)-\frac{5 \pi^{2}}{3}\right]+\mathcal{O}(\epsilon), \\
I_{3} & =\frac{c_{\Gamma}}{2 \beta s_{12}}\left(\frac{s_{12}}{\mu^{2}}\right)^{-\epsilon}\left[\log \left(x_{S}\right)\left(\frac{1}{\epsilon}-\frac{1}{2} \log \left(x_{S}\right)-2 \log (\beta)\right)-2 \operatorname{Li}_{2}\left(x_{S}\right)+\frac{\pi^{2}}{3}\right]+\mathcal{O}(\epsilon) .
\end{aligned}
$$

The dual integral $I_{3}$ is purely real, while the dual integral $I_{2}$ generates an imaginary component due to the intersection of the forward on-shell hyperboloid of $G_{\mathrm{F}}\left(q_{2}\right)$ with the backward on-shell hyperboloid of $G_{\mathrm{F}}\left(q_{3}\right)$. Its imaginary part can be calculated to all orders in $\epsilon$ from

$$
\begin{aligned}
\imath \operatorname{Im}\left(I_{2}\right) & =\frac{1}{2} \int_{\ell} G_{\mathrm{D}}\left(q_{2} ; q_{1}\right) \tilde{\delta}\left(q_{2}\right) \tilde{\delta}\left(-q_{3}\right) \\
& =-\imath \pi \frac{2}{s_{12}} \int \frac{\delta\left(1-\xi_{2,0}\right) \xi_{2}^{2} d\left[\xi_{2}\right] d\left[v_{2}\right]}{\xi_{2,0}\left(\xi_{2,0}+\beta \xi_{2}\left(1-2 v_{2}\right)-m^{2}\right)}=\imath \frac{\widetilde{c}_{\Gamma}}{\beta s_{12}}\left(\frac{\beta^{2} s_{12}}{\mu^{2}}\right)^{-\epsilon} \frac{\sin (2 \pi \epsilon)}{2 \epsilon^{2}},
\end{aligned}
$$

which is the expected result obtained through the application of the Cutkosky's rule. The sum of these contributions in eq. (3.19) and eq. (3.20) agrees with the original Feynman integral in eq. (3.2).

\section{Massive scalar decay rate in DREG}

In order to establish a physical parallelism and understand the subtraction of IR singularities, we work in a simplified toy scalar model with a massive scalar particle $\phi$ which couples 

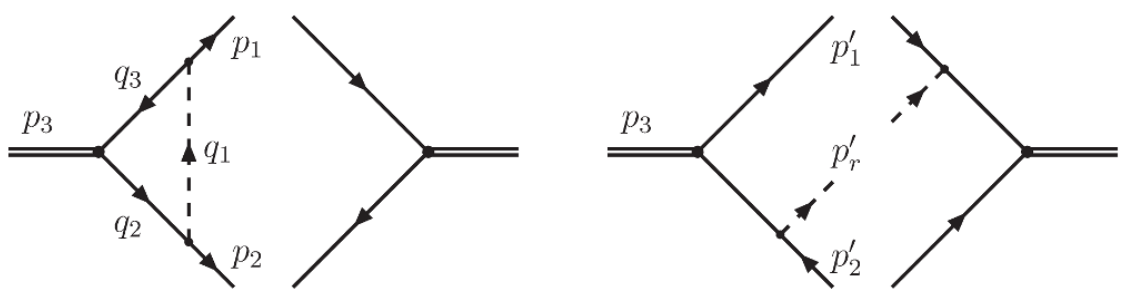

Figure 3. Kinematic configuration of the NLO corrections to the decay process $\phi \rightarrow \phi+\phi$. The one-loop contribution is proportional to the scalar three-point function, with a virtual massless $\psi$ inside the loop (left). The real contribution is due to the interference terms originated by the emission of an on-shell massless particle $\psi$ (right). In this case, the momentum configuration is given by $p_{3} \rightarrow p_{1}^{\prime}+p_{2}^{\prime}+p_{r}^{\prime}$.

to a massless one, $\psi$. In concrete, we consider the decay process $\phi\left(p_{3}\right) \rightarrow \phi\left(p_{1}\right)+\phi\left(p_{2}\right)$, with $p_{1}^{2}=M^{2}=p_{2}^{2}$ (on-shell massive external particles) and $p_{3}^{2}=s_{12}$ (off-shell incoming particle). The Born-level decay rate is given by

$$
\Gamma^{(0)}=\frac{g^{2}}{2 \sqrt{s_{12}}} \int d \Phi_{1 \rightarrow 2},
$$

where $g$ is the coupling and $s_{12}>4 M^{2}$ to guarantee the physical feasibility of the process. To compute the corresponding NLO correction, we need to add virtual (i.e. one-loop) and real (i.e. extra-radiation) contributions. We will assume the presence of only one massless particle inside the loop, as well as the emission of a massless real particle in the extraradiation contribution. The corresponding NLO diagrams are exhibited in figure $3 .^{2}$

Let's start with the virtual part, which we assume proportional to the scalar three-point function, i.e.

$$
\Gamma_{\mathrm{V}}^{(1)}=\frac{1}{2 \sqrt{s_{12}}} \int d \Phi_{1 \rightarrow 2} 2 \operatorname{Re}\left\langle\mathcal{M}^{(0)} \mid \mathcal{M}^{(1)}\right\rangle=-\Gamma^{(0)} 2 g^{2} s_{12} \operatorname{Re} L_{m>0}^{(1)}\left(p_{1}, p_{2},-p_{3}\right) .
$$

Since $s_{12}>0$, the virtual decay rate is given from eq. (3.19) as a function of $x_{S}$

$$
\Gamma_{\mathrm{V}}^{(1)}=\Gamma^{(0)} \frac{c_{\Gamma} 4 g^{2}}{\beta}\left(\frac{s_{12}}{\mu^{2}}\right)^{-\epsilon}\left[\log \left(x_{S}\right)\left(-\frac{1}{2 \epsilon}+\frac{1}{4} \log \left(x_{S}\right)+\log (\beta)\right)+\operatorname{Li}_{2}\left(x_{S}\right)+\frac{\pi^{2}}{3}\right]+\mathcal{O}(\epsilon) .
$$

In order to calculate the totally inclusive decay rate, we have to consider the real emission process. In this particular toy-example the cancellation of IR-singularities is achieved by including only the interference terms originated in the process $\phi\left(p_{3}\right) \rightarrow \phi\left(p_{1}^{\prime}\right)+\phi\left(p_{2}^{\prime}\right)+\psi\left(p_{r}^{\prime}\right)$, as depicted schematically in figure 3. Explicitly,

$$
\begin{aligned}
\Gamma_{\mathrm{R}}^{(1)}=\frac{1}{2 \sqrt{s_{12}}} \int d \Phi_{1 \rightarrow 3} 2 \operatorname{Re}\left\langle\mathcal{M}_{2 r}^{(0)} \mid \mathcal{M}_{1 r}^{(0)}\right\rangle & =\frac{g^{4}}{2 \sqrt{s_{12}}} \int d \Phi_{1 \rightarrow 3} \frac{2 s_{12}}{\left(2 p_{1}^{\prime} \cdot p_{r}^{\prime}\right)\left(2 p_{2}^{\prime} \cdot p_{r}^{\prime}\right)} \\
& =\Gamma^{(0)} 2 g^{2} \frac{(4 \pi)^{\epsilon-2}}{\Gamma(1-\epsilon)}\left(\frac{s_{12}}{\mu^{2}}\right)^{-\epsilon} \beta^{-1+2 \epsilon} \int \theta\left(h_{p}\right) h_{p}^{-\epsilon} \frac{d y_{1 r}^{\prime} d y_{2 r}^{\prime}}{y_{1 r}^{\prime} y_{2 r}^{\prime}},
\end{aligned}
$$

\footnotetext{
${ }^{2}$ This decay rate does not correspond to any physical theory, since the full set of Feynman diagrams has not be taken into account. However, for illustrative purposes, it is enough to restrict the following discussion to virtual and real contributions with a similar topology [36, 39].
} 
where we used the definition of the massive three-body phase space in eq. (A.2) and $y_{i r}^{\prime}=2 p_{i}^{\prime} \cdot p_{r}^{\prime} / s_{12}$. To compute this integral, we apply the change of variables suggested in appendix A, which allows to factorise the energy and the angular dependence of the integrand. By using eq. (A.4), we obtain

$$
\begin{aligned}
\Gamma_{\mathrm{R}}^{(1)}= & \Gamma^{(0)} 2 g^{2} \frac{(4 \pi)^{\epsilon-2}}{\Gamma(1-\epsilon)}\left(\frac{s_{12}}{\mu^{2}}\right)^{-\epsilon} \beta^{-1+2 \epsilon}\left(1+x_{S}\right)^{6 \epsilon} \\
& \times \int_{x_{S}}^{x_{S}^{-1}} d z \frac{z^{-1+2 \epsilon}(1+z)^{2 \epsilon}}{\left(z-x_{S}\right)^{3 \epsilon}\left(1-x_{S} z\right)^{3 \epsilon}} \int_{0}^{1} d w w^{-1-2 \epsilon}(1-w)^{-\epsilon}
\end{aligned}
$$

The integration in $w$ can be trivially performed, and it leads to the appearance of an $\epsilon$-pole. The integral in $z$ is finite if $x_{S}>0$ (i.e. in the massive case), thus we expand the integrand in $\epsilon$ before the integration. The resulting expression is

$$
\begin{aligned}
\Gamma_{\mathrm{R}}^{(1)}= & \Gamma^{(0)} \frac{c_{\Gamma} 4 g^{2}}{\beta}\left(\frac{s_{12}}{\mu^{2}}\right)^{-\epsilon}\left[\log \left(x_{S}\right)\left(\frac{1}{2 \epsilon}-\frac{1}{4} \log \left(x_{S}\right)+\log \left(1+x_{S}\right)+\log \left(1-x_{S}^{2}\right)\right)\right. \\
& \left.+\operatorname{Li}_{2}\left(x_{S}\right)+\operatorname{Li}_{2}\left(x_{S}^{2}\right)-\frac{\pi^{2}}{3}\right]+\mathcal{O}(\epsilon) .
\end{aligned}
$$

Putting together the virtual and real contributions from eq. (4.3) and eq. (4.6), we get

$$
\Gamma^{(1)}=\Gamma^{(0)} \frac{4 a}{\beta}\left[\log \left(x_{S}\right)\left(\log \left(1-x_{S}\right)+\log \left(1-x_{S}^{2}\right)\right)+2 \operatorname{Li}_{2}\left(x_{S}\right)+\operatorname{Li}_{2}\left(x_{S}^{2}\right)\right]+\mathcal{O}(\epsilon),
$$

with $a=g^{2} /(4 \pi)^{2}$. The purpose of the following discussion will be the derivation of a purely four-dimensional representation of this result, through the local cancellation of all the IR divergences present in both real and virtual contributions. It is crucial to emphasise that this cancellation of IR singularities at integrand level is achieved by a suitable mapping of momenta.

\section{Phase-space partition and real-virtual mapping with massive particles}

The first ingredient that we need to introduce is a complete partition of the real phasespace $[36,39]$, in such a way that each individual region of that partition contains a single soft, collinear or quasi-collinear configuration. The quasi-collinear configurations are those in which a massless particle becomes collinear with a massive one [23]. In that case the mass acts as an IR-regulator and the collinear $\epsilon$-poles that appear in DREG in the massless case are transformed into finite logarithmic terms in the mass. These logarithmic contributions are cancelled in the total cross-section but the massless and the $\epsilon \rightarrow 0$ limits do not commute for the virtual and real corrections separately. Thus, we split the real phasespace by defining the domains

$$
\mathcal{R}_{i}=\left\{y_{i r}^{\prime}<\min \left(y_{j k}^{\prime}\right)\right\}, \quad \sum \mathcal{R}_{i}=1 .
$$

For instance, $\mathcal{R}_{i}$ selects configurations with $p_{i}^{\prime} \| p_{r}^{\prime}$ or close to collinear, excluding the remaining ones. In particular, this partition reduces to

$$
\theta\left(y_{2 r}^{\prime}-y_{1 r}^{\prime}\right)+\theta\left(y_{1 r}^{\prime}-y_{2 r}^{\prime}\right)=1
$$


for the simplest $1 \rightarrow 3$ scenario. The definition of the phase-space partition in eq. (5.1) is the same that we would use in the massless case [39]; the only difference is that now some of the external momenta are massive. That partition, together with the well-motivated mapping of momenta that will be presented in the following, ensures a smooth massless limit, therefore an integrand level cancellation of the logarithmic dependences in the mass arising from the quasi-collinear configurations of the virtual and real corrections, and thus a more stable numerical implementation of the method.

Then, we shall define a proper momentum mapping in each region to match the singular behaviour of the real and the dual integrands. In order to properly combine real and virtual contributions at integrand level, we need to generate the $N+1$ on-shell kinematics by making use of the $N$-parton Born-level process and the on-shell loop momenta. One of the main difficulties in constructing a momentum mapping with massive particles is that the on-shell conditions lead to quadratic equations in the mapping parameters. However, it is very well-known that massive vectors can be expressed in terms of two massless momenta. Then, we can exploit this property to simplify the mapping equations. For the case of a pair of particles of the same mass, the corresponding massive momenta can be written as

$$
p_{1}^{\mu}=\beta_{+} \hat{p}_{1}^{\mu}+\beta_{-} \hat{p}_{2}^{\mu}, \quad p_{2}^{\mu}=\beta_{-} \hat{p}_{1}^{\mu}+\beta_{+} \hat{p}_{2}^{\mu},
$$

with $\hat{p}_{1}^{2}=\hat{p}_{2}^{2}=0$ and $\beta_{ \pm}=(1 \pm \beta) / 2$. Moreover, the massless momenta fulfil the following useful identities

$$
2 \hat{p}_{1} \cdot \hat{p}_{2}=s_{12}, \quad \hat{p}_{1}^{\mu}+\hat{p}_{2}^{\mu}=p_{1}^{\mu}+p_{2}^{\mu} .
$$

In their centre-of-mass frame, these massless momenta are simply given by

$$
\hat{p}_{1}^{\mu}=\frac{\sqrt{s_{12}}}{2}\left(1, \mathbf{0}_{\perp}, 1\right), \quad \hat{p}_{2}^{\mu}=\frac{\sqrt{s_{12}}}{2}\left(1, \mathbf{0}_{\perp},-1\right) .
$$

Going back to the toy example in section 4, let's start with the first region where $\mathcal{R}_{1}=1$ (i.e. $y_{1 r}^{\prime}<y_{2 r}^{\prime}$ ). Motivated by the factorisation properties of QCD in the quasicollinear limit and the momentum decomposition in eq. (5.3), we propose the following mapping with $q_{1}^{2}=0$

$$
\begin{aligned}
& p_{r}^{\prime \mu}=q_{1}^{\mu}, \\
& p_{1}^{\prime \mu}=\left(1-\alpha_{1}\right) \hat{p}_{1}^{\mu}+\left(1-\gamma_{1}\right) \hat{p}_{2}^{\mu}-q_{1}^{\mu}, \\
& p_{2}^{\prime \mu}=\alpha_{1} \hat{p}_{1}^{\mu}+\gamma_{1} \hat{p}_{2}^{\mu},
\end{aligned}
$$

which fulfils the momentum conservation constraint by construction. As in the massless case, the momentum $p_{2}^{\prime}$ acts as the spectator of the splitting process and is used to balance momentum conservation. The emitters have momenta $p_{1}$ and $p_{1}^{\prime}$, and have the same mass. Although restricted to three final-state particles, the momentum mapping in eq. (5.6) can easily be generalised to the multipartonic case, with $p_{k}^{\prime}=p_{k}$ for $k \neq 1,2, r$. The parameters $\alpha_{1}$ and $\gamma_{1}$ are determined from the two on-shell conditions

$$
\begin{aligned}
& \left(p_{1}^{\prime}\right)^{2}=\left(1-\alpha_{1}\right)\left(1-\gamma_{1}\right) s_{12}-2 q_{1} \cdot\left(\left(1-\alpha_{1}\right) \hat{p}_{1}+\left(1-\gamma_{1}\right) \hat{p}_{2}\right)=M^{2}, \\
& \left(p_{2}^{\prime}\right)^{2}=\alpha_{1} \gamma_{1} s_{12}=M^{2}
\end{aligned}
$$


whose explicit solutions are

$$
\begin{aligned}
\alpha_{1} & =\frac{1-\xi_{1,0}-\sqrt{\left(1-\xi_{1,0}\right)^{2}-m^{2}\left(1-\xi_{1,0}+v_{1}\left(1-v_{1}\right) \xi_{1,0}^{2}\right)}}{2\left(1-v_{1} \xi_{1,0}\right)}, \\
\gamma_{1} & =\frac{1-\xi_{1,0}+\sqrt{\left(1-\xi_{1,0}\right)^{2}-m^{2}\left(1-\xi_{1,0}+v_{1}\left(1-v_{1}\right) \xi_{1,0}^{2}\right)}}{2\left(1-\left(1-v_{1}\right) \xi_{1,0}\right)}
\end{aligned}
$$

whilst $\left(p_{r}^{\prime}\right)^{2}=0$ by construction, since $q_{1}^{2}=0$. Due to the fact that we are dealing with quadratic equations, there are two sets of solutions. The solution in eq. (5.8) is compatible with the soft limit; it recovers the Born-level kinematics when $\xi_{1,0} \rightarrow 0$. In that limit, $\left(\alpha_{1}, \gamma_{1}\right) \rightarrow\left(\beta_{-}, \beta_{+}\right)$and therefore $\left(p_{1}^{\prime}, p_{2}^{\prime}\right) \rightarrow\left(p_{1}, p_{2}\right)$. Also, it properly reduces to the massless parametrisation defined in refs. [36, 39], i.e. if $m \rightarrow 0$, we have

$$
\alpha_{1} \rightarrow 0, \quad \gamma_{1} \rightarrow \frac{1-\xi_{1,0}}{1-\left(1-v_{1}\right) \xi_{1,0}} .
$$

Using these definitions, the kinematical invariants $y_{i j}^{\prime}$ become

$$
\begin{aligned}
y_{1 r}^{\prime} & =\frac{\xi_{1,0}}{1-\left(1-v_{1}\right) \xi_{1,0}}\left(v_{1}+\alpha_{1}\left(1-2 v_{1}\right)\right) \\
y_{2 r}^{\prime} & =\frac{\xi_{1,0}}{1-\left(1-v_{1}\right) \xi_{1,0}}\left(\left(1-v_{1}\right)\left(1-\xi_{1,0}\right)-\alpha_{1}\left(1-2 v_{1}\right)\right), \\
y_{12}^{\prime} & =1-\xi_{1,0}-\frac{m^{2}}{2}
\end{aligned}
$$

which fullfil $y_{12}^{\prime}+y_{1 r}^{\prime}+y_{2 r}^{\prime}=1-m^{2} / 2$. Again, we recover easily the massless expressions [36, $39]$ with $\alpha_{1}=0$. In order to improve the presentation of the results, it is also convenient to express the mass in terms of $\alpha_{1}$,

$$
m^{2}=\frac{4 \alpha_{1}\left(1-\xi_{1,0}-\alpha_{1}\left(1-v_{1} \xi_{1,0}\right)\right)}{1-\left(1-v_{1}\right) \xi_{1,0}} .
$$

Then, we compute the associated Jacobian in the physically allowed region (i.e. those points belonging to the domain $\mathcal{R}_{1}$ ), which is given by

$$
\mathcal{J}_{1}\left(\xi_{1,0}, v_{1}\right)=\frac{\xi_{1,0}\left(1-\xi_{1,0}-\alpha_{1}\left(2-\xi_{1,0}\right)\right)^{2}}{\left(1-\left(1-v_{1}\right) \xi_{1,0}\right)^{2}\left(1-\xi_{1,0}-2 \alpha_{1}\left(1-v_{1} \xi_{1,0}\right)\right)},
$$

with $d y_{1 r}^{\prime} d y_{2 r}^{\prime}=\mathcal{J}_{1}\left(\xi_{1,0}, v_{1}\right) d \xi_{1,0} d v_{1}$. Notice that this expression is apparently free of square roots, since the mass dependence was rewritten in terms of $\alpha_{1}$, as suggested in eq. (5.11).

On the other hand, we have to express $\mathcal{R}_{1}$ in terms of the dual variables. If we use the mapping given in eq. (5.6), we obtain

$$
\mathcal{R}_{1}\left(\xi_{1,0}, v_{1}\right)=\theta\left(1-2 v_{1}\right) \theta\left(\frac{1-2 v_{1}}{1-v_{1}}\left(1-\frac{1-\sqrt{1-4 m^{2} v_{1}\left(1-v_{1}\right)}}{2 v_{1}}\right)-\xi_{1,0}\right)
$$




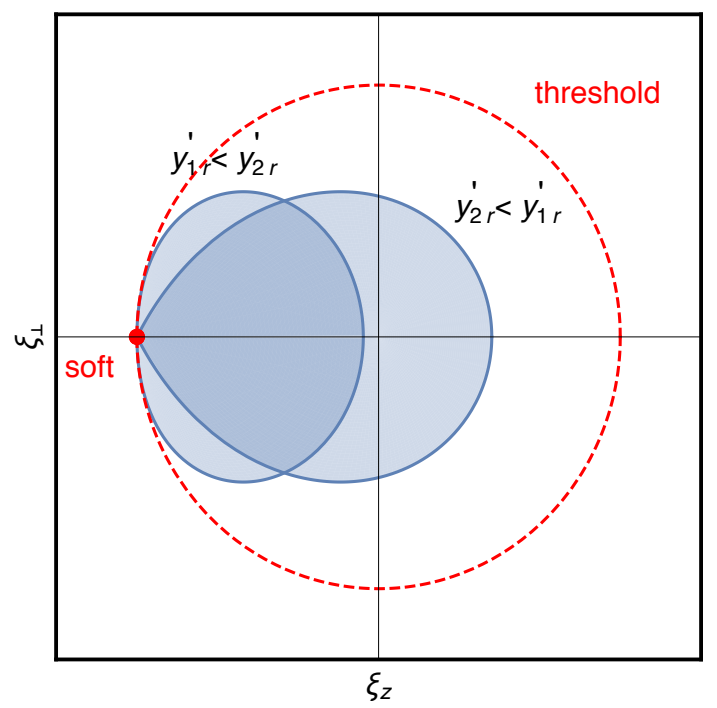

Figure 4. The dual integration regions in the loop three-momentum space, with $\xi_{\perp}=\sqrt{\xi_{x}^{2}+\xi_{y}^{2}}$.

which is the characteristic function associated to the domain $\mathcal{R}_{1}$. In figure 4 , we show this domain in the loop three-momentum space. The massless limit agrees with the expected result. Moreover, the three-body phase-space limits defined by the condition $h_{p}=0$ are simply determined by $v_{1}=0$.

In the complementary region, where $\mathcal{R}_{2}=1$ (i.e. $y_{2 r}^{\prime}<y_{1 r}^{\prime}$ ) with $q_{2}^{2}=M^{2}$, the mapping is defined by ${ }^{3}$

$$
\begin{aligned}
& p_{r}^{\prime \mu}=\left(1-\gamma_{2}\right) \hat{p}_{1}^{\mu}+\left(1-\alpha_{2}\right) \hat{p}_{2}^{\mu}-q_{2}^{\mu}, \\
& p_{1}^{\prime \mu}=\gamma_{2} \hat{p}_{1}^{\mu}+\alpha_{2} \hat{p}_{2}^{\mu}, \\
& p_{2}^{\prime \mu}=q_{2}^{\mu}
\end{aligned}
$$

where

$$
\begin{aligned}
& \left(p_{1}^{\prime}\right)^{2}=\alpha_{2} \gamma_{2} s_{12}=M^{2}, \\
& \left(p_{r}^{\prime}\right)^{2}=M^{2}+\left(1-\alpha_{2}\right)\left(1-\gamma_{2}\right) s_{12}-2 q_{2} \cdot\left(\left(1-\gamma_{2}\right) \hat{p}_{1}+\left(1-\alpha_{2}\right) \hat{p}_{2}\right)=0,
\end{aligned}
$$

are the associated on-shell conditions. In this case, the condition $\left(p_{2}^{\prime}\right)^{2}=M^{2}$ is fulfilled by construction. Solving the system and selecting the physical solution, we get

$$
\begin{aligned}
\alpha_{2} & =\frac{1-\xi_{2,0}+m^{2} / 2-\sqrt{\left(1-\xi_{2,0}\right)^{2}-m^{2} v_{2}\left(1-v_{2}\right) \xi_{2}^{2}}}{2-\left(1-2 v_{2}\right) \xi_{2}-\xi_{2,0}} \\
\gamma_{2} & =\frac{1-\xi_{2,0}+m^{2} / 2+\sqrt{\left(1-\xi_{2,0}\right)^{2}-m^{2} v_{2}\left(1-v_{2}\right) \xi_{2}^{2}}}{2+\left(1-2 v_{2}\right) \xi_{2}-\xi_{2,0}}
\end{aligned}
$$

In order to check the consistency of this solution, we consider the massless limit, obtaining

$$
\alpha_{2} \rightarrow 0, \quad \gamma_{2} \rightarrow \frac{1-\xi_{2}}{1-v_{2} \xi_{2}},
$$

\footnotetext{
${ }^{3}$ We have exchanged the role of the radiated particle and the emitter to keep $p_{2}^{\prime}$ massive.
} 
which implies that the parametrisation reduces to the expected one. On the other hand, the two-body invariants are given by

$$
\begin{aligned}
& y_{1 r}^{\prime}=1-\xi_{2,0}, \\
& y_{2 r}^{\prime}=\frac{\xi_{2,0}+\left(1-2 v_{2}\right)\left(1-2 \alpha_{2}\right) \xi_{2}-m^{2}}{2+\left(1-2 v_{2}\right) \xi_{2}-\xi_{2,0}}, \\
& y_{12}^{\prime}=\xi_{2,0}-\frac{m^{2}}{2}-y_{2 r}^{\prime},
\end{aligned}
$$

and, since this mapping will be used in the region of the real phase-space defined by $\mathcal{R}_{2}$, we rewrite the associated characteristic function as

$$
\mathcal{R}_{2}\left(\xi_{2}, v_{2}\right)=\theta\left(\beta^{2}\left(\left(1+\sqrt{\left(1-v_{2}\right)\left(1-m^{2} v_{2}\right)}\right)^{2}-m^{2} v_{2}^{2}\right)^{-1 / 2}-\xi_{2}\right)
$$

The corresponding domain in the loop three-momentum space is also shown in figure 4 . The associated Jacobian is given by

$\mathcal{J}_{2}\left(\xi_{2}, v_{2}\right)=\frac{4 \xi_{2}^{2}\left(1-\xi_{2,0}+m^{2} / 2-\alpha_{2}\left(2-\xi_{2,0}\right)\right)^{2}}{\xi_{2,0}\left(2+\left(1-2 v_{2}\right) \xi_{2}-\xi_{2,0}\right)^{2}\left(1-\xi_{2,0}+m^{2} / 2-\alpha_{2}\left(2-\left(1-2 v_{2}\right) \xi_{2}-\xi_{2,0}\right)\right)}$

with $d y_{1 r}^{\prime} d y_{2 r}^{\prime}=\mathcal{J}_{2}\left(\xi_{2}, v_{2}\right) d \xi_{2} d v_{2}$, and we made use of the identity

$$
m^{2}=\frac{4 \alpha_{2}\left(2\left(1-\xi_{2,0}\right)-\alpha_{2}\left(2-\left(1-2 v_{2}\right) \xi_{2}-\xi_{2,0}\right)\right)}{2+\left(1-2 v_{2}\right) \xi_{2}-\xi_{2,0}-4 \alpha_{2}},
$$

to simplify the expressions.

\subsection{General momentum mapping}

The momentum mappings previously presented can easily be extended to the most general multipartonic case in which the emitter and the spectator have different masses: $p_{i}^{2}=m_{i}^{2}$ and $p_{j}^{2}=m_{j}^{2}$, respectively. The decomposition of their momenta in terms of two massless momenta $\left(\hat{p}_{i}^{2}=\hat{p}_{j}^{2}=0\right)$ is given by

$$
\begin{aligned}
p_{i}^{\mu} & =\beta_{+} \hat{p}_{i}^{\mu}+\beta_{-} \hat{p}_{j}^{\mu}, \\
p_{j}^{\mu} & =\left(1-\beta_{+}\right) \hat{p}_{i}^{\mu}+\left(1-\beta_{-}\right) \hat{p}_{j}^{\mu},
\end{aligned}
$$

with

$$
\beta_{ \pm}=\frac{s_{i j}+m_{i}^{2}-m_{j}^{2} \pm \lambda\left(s_{i j}, m_{i}^{2}, m_{j}^{2}\right)}{2 s_{i j}},
$$

where $\lambda\left(s_{i j}, m_{i}^{2}, m_{j}^{2}\right)=\sqrt{\left(s_{i j}-\left(m_{i}-m_{j}\right)^{2}\right)\left(s_{i j}-\left(m_{i}+m_{j}\right)^{2}\right)}$ is the usual Kallén function. The massless momenta fulfil the useful condition $\hat{p}_{i}+\hat{p}_{j}=p_{i}+p_{j}$. The mapping 
with the momenta of the real process is formally equal to the mapping already considered in eq. (5.6), i.e.

$$
\begin{array}{ll}
p_{r}^{\prime \mu}=q_{i}^{\mu}, & \\
p_{i}^{\prime \mu}=\left(1-\alpha_{i}\right) \hat{p}_{i}^{\mu}+\left(1-\gamma_{i}\right) \hat{p}_{j}^{\mu}-q_{i}^{\mu}, & \\
p_{j}^{\prime \mu}=\alpha_{i} \hat{p}_{i}^{\mu}+\gamma_{i} \hat{p}_{j}^{\mu}, & \\
p_{k}^{\prime \mu}=p_{k}^{\mu}, & k \neq i, j, r .
\end{array}
$$

It leads to the on-shell conditions

$$
\begin{aligned}
& \left(p_{i}^{\prime}\right)^{2}=\left(1-\alpha_{i}\right)\left(1-\gamma_{i}\right) s_{i j}-2 q_{i} \cdot\left(\left(1-\alpha_{i}\right) \hat{p}_{i}+\left(1-\gamma_{i}\right) \hat{p}_{j}\right)+m_{r}^{2}=\left(m_{i}^{\prime}\right)^{2}, \\
& \left(p_{j}^{\prime}\right)^{2}=\alpha_{i} \gamma_{i} s_{i j}=m_{j}^{2} .
\end{aligned}
$$

In eq. (5.25), we have imposed that the spectator and the radiated particle have the same flavour (and, thus, the same mass) in the virtual and real processes; $p_{j}^{2}=\left(p_{j}^{\prime}\right)^{2}=m_{j}^{2}$ and $q_{i}^{2}=\left(p_{r}^{\prime}\right)^{2}=m_{r}^{2}$, respectively. The emitter, however, might change flavour, $\left(p_{i}^{\prime}\right)^{2}=\left(m_{i}^{\prime}\right)^{2} \neq$ $m_{i}^{2}$. This situation occurs, for instance, when a gluon splits into a massive quark-antiquark pair. The solution to eq. (5.25) for the parameters of the mapping reads

$$
\begin{aligned}
\alpha_{i} & =\frac{\left(p_{i j}-q_{i}\right)^{2}+m_{j}^{2}-\left(m_{i}^{\prime}\right)^{2}-\Lambda_{i j}}{2\left(s_{i j}-2 q_{i} \cdot \hat{p}_{i}\right)}, \\
\gamma_{i} & =\frac{\left(p_{i j}-q_{i}\right)^{2}+m_{j}^{2}-\left(m_{i}^{\prime}\right)^{2}+\Lambda_{i j}}{2\left(s_{i j}-2 q_{i} \cdot \hat{p}_{j}\right)},
\end{aligned}
$$

with

$$
\Lambda_{i j}=\sqrt{\left(\left(p_{i j}-q_{i}\right)^{2}+m_{j}^{2}-\left(m_{i}^{\prime}\right)^{2}\right)^{2}-\frac{4 m_{j}^{2}}{s_{i j}}\left(s_{i j}-2 q_{i} \cdot \hat{p}_{i}\right)\left(s_{i j}-2 q_{i} \cdot \hat{p}_{j}\right)} .
$$

The momentum mapping in eq. (5.24) has an smooth limit whenever any of the involved particles becomes massless; in particular, if the spectator is a massless particle, then $\alpha_{i}=0$.

\section{Massive scalar decay rate from four-dimensional unsubstraction}

In this section we illustrate the method of LTD four-dimensional unsubstraction [36, 39] with the massive toy example presented in section 4. All the necessary ingredients have been presented in the previous sections. We combine at integrand level the dual loop contributions (section 3) with the real-radiation terms (section 4) with the help of the momentum mappings defined in section 5. Since the sum of all the contributions is UV and IR finite, the final result is free of $\epsilon$-poles. We would like to emphasise that, in a generic situation, this assertion is not enough to guarantee the integrability of the expressions in four-dimensions. However, by virtue of the momentum mappings and the unification of the dual coordinates, LTD leads naturally to a local cancellation of divergences and the limit $\epsilon \rightarrow 0$ can be considered at integrand level. 
The LTD representation of the virtual decay rate in this toy example is given by

$$
\Gamma_{\mathrm{V}}^{(1)}=\frac{1}{2 \sqrt{s_{12}}} \sum_{i=1}^{3} \int d \Phi_{1 \rightarrow 2} 2 \operatorname{Re}\left\langle\mathcal{M}^{(0)} \mid \mathcal{M}^{(1)}\left(\tilde{\delta}\left(q_{i}\right)\right)\right\rangle,
$$

with

$$
\left\langle\mathcal{M}^{(0)} \mid \mathcal{M}^{(1)}\left(\tilde{\delta}\left(q_{i}\right)\right)\right\rangle=-g^{4} s_{12} I_{i}
$$

where the dual integrals $I_{i}$ are defined in eq. (3.13), and we take the integration measure exactly with $\epsilon=0$. In order to ensure the cross-cancellation of spurious singularities and get a direct $\epsilon=0$ limit we must rewrite all the on-shell momenta in terms of the same coordinate system. This change of variables is explained in appendix B. With that change of variables the virtual decay rate in eq. (6.1) becomes a single unconstrained integral in the loop three-momentum.

We also consider the real contribution given by eq. (4.4). First, we split the real three-body phase-space according to eq. (5.2), and define

$$
\widetilde{\Gamma}_{\mathrm{R}, i}^{(1)}=\frac{1}{2 \sqrt{s_{12}}} \int d \Phi_{1 \rightarrow 3} 2 \operatorname{Re}\left\langle\mathcal{M}_{2 r}^{(0)} \mid \mathcal{M}_{1 r}^{(0)}\right\rangle \mathcal{R}_{i}\left(y_{i r}^{\prime}<y_{j r}^{\prime}\right), \quad i, j=\{1,2\}
$$

that obviously fulfill

$$
\Gamma_{\mathrm{R}}^{(1)}=\widetilde{\Gamma}_{\mathrm{R}, 1}^{(1)}+\widetilde{\Gamma}_{\mathrm{R}, 2}^{(1)}
$$

Second, in each region of the real phase-space we apply one of the momentum mappings defined in eq. (5.6) and eq. (5.14), respectively. The main advantage of these mappings is that they are optimised to deal smoothly with the massless limit in each of the two regions. Thus, we rewrite the real contributions in terms of the loop variables and we obtain

$$
\begin{aligned}
& \widetilde{\Gamma}_{\mathrm{R}, 1}^{(1)}=\Gamma^{(0)} \frac{2 a}{\beta} \int d \xi_{1,0} d v_{1} \frac{\mathcal{R}_{1}\left(\xi_{1,0}, v_{1}\right) \mathcal{J}_{1}\left(\xi_{1,0}, v_{1}\right)\left(1-\xi_{1,0}\left(1-v_{1}\right)\right)^{2}}{\xi_{1,0}^{2}\left(v_{1}+\alpha_{1}\left(1-2 v_{1}\right)\right)\left(\left(1-v_{1}\right)\left(1-\xi_{1,0}\right)-\alpha_{1}\left(1-2 v_{1}\right)\right)} \\
& \widetilde{\Gamma}_{\mathrm{R}, 2}^{(1)}=\Gamma^{(0)} \frac{2 a}{\beta} \int d \xi_{2} d v_{2} \frac{\mathcal{R}_{2}\left(\xi_{2}, v_{2}\right) \mathcal{J}_{2}\left(\xi_{2}, v_{2}\right)\left(2+\left(1-2 v_{2}\right) \xi_{2}-\xi_{2,0}\right)}{\left(1-\xi_{2,0}\right)\left(\xi_{2,0}+\left(1-2 v_{2}\right)\left(1-2 \alpha_{2}\right) \xi_{2}-m^{2}\right)}
\end{aligned}
$$

where the Jacobians of the respective transformations are given by eq. (5.12) and eq. (5.20), and the integration domains, which are restricted by the functions $\mathcal{R}_{i}$ from eq. (5.13) and eq. (5.19), are shown in figure 4. Again, after applying the change of variables defined in appendix B to bring the two real contributions to a common coordinate system, we can consistently take the limit $\epsilon=0$, directly at integrand level.

The sum of all the virtual and real contributions, eqs. (6.1), (6.5) and (6.6), is a finite function in the $\epsilon=0$ limit because all the IR singularities are cancelled locally in the loop three-momentum space at integrand level. The virtual contribution, however, contains a threshold singularity at $\xi_{2}=\beta$. This singularity is integrable and can be treated numerically by contour deformation [33-35]. For the toy scalar model and the physical examples that we are considering in this article there is a simplest solution: we 


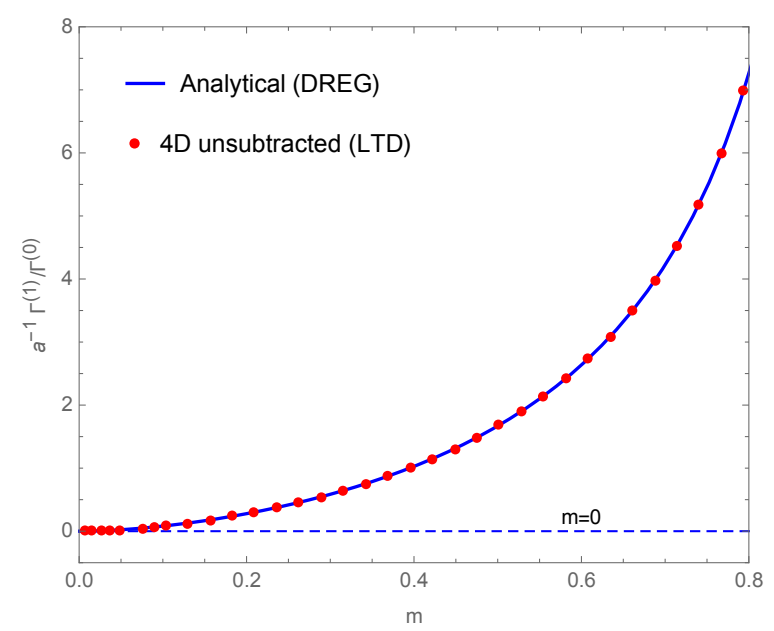

Figure 5. Total decay rate at NLO normalised to the leading order for the toy scalar example, $a^{-1} \Gamma^{(1)} / \Gamma^{(0)}$, as a function of the dimensionless mass parameter $m$. The horizontal dashed line represents the massless limit, the solid line corresponds to the analytic result obtained through DREG, and the dots are obtained numerically through LTD unsubtraction.

can compactify the high-energy region with $\xi>\beta$ into the unit sphere by using a change of variables. Explicitly, since the integrand is a function of the modulus of the threemomentum and the polar angle, then

$$
\int_{0}^{\infty} d \xi g(\xi, v)=\beta \int_{0}^{1} d x\left[g(\beta x, v)+x^{-2} g\left(\beta x^{-1}, v\right)\right]
$$

where the threshold singularity has been mapped into to the upper end-point, namely $x=1$. This approach is very efficient for the numerical implementation.

Finally, we numerically integrate simultaneously the virtual and real corrections from eqs. (6.1), (6.5) and (6.6) with the help of eq. (6.7) to obtain the total decay rate at NLO, $\Gamma^{(1)}$, as a function of the dimensionless mass parameter $m$. The result is shown in figure 5 , and it is compared with the DREG analytic expression given by eq. (4.7). The agreement is excellent and quite stable numerically. Computing all the points of the plot in figure 5 takes a few minutes in a standard laptop $(2.3 \mathrm{GHz}$ quad-core processor). Moreover, the massless transition is very smooth because the momentum mappings are optimised to deal with the quasi-collinear configurations. In other words, the massless limit can directly be taken at the integrand level. This is another interesting advantage of the LTD approach.

\section{Unintregrated wave function and mass renormalisation for heavy quarks}

In order to consider physical processes with heavy quarks we should also take into account self-energy corrections. The well-known expressions of the wave function and mass 
renormalisation constants, in the Feynman gauge with on-shell renormalisation conditions,

$$
\begin{aligned}
\Delta Z_{2} & =\frac{\alpha_{\mathrm{S}}}{4 \pi} C_{\mathrm{F}}\left(-\frac{1}{\epsilon_{\mathrm{UV}}}-\frac{2}{\epsilon_{\mathrm{IR}}}+3 \log \left(\frac{M^{2}}{\mu^{2}}\right)-4\right), \\
\Delta Z_{M}^{\mathrm{OS}} & =\frac{\alpha_{\mathrm{S}}}{4 \pi} C_{\mathrm{F}}\left(-\frac{3}{\epsilon_{\mathrm{UV}}}+3 \log \left(\frac{M^{2}}{\mu^{2}}\right)-4\right),
\end{aligned}
$$

are not suitable, in particular, for the implementation of a local subtraction of the IR singularities. We shall provide unintegrated expressions. The case of massless quarks has been studied in detail in ref. [39]. In eq. (7.1), we explicitly identify the origin of the $\epsilon$-poles, and the IR singularities of the wave function should cancel the IR singularities arising from the squared amplitudes of the real processes with radiated gluons off quarks.

We consider the process in which there are two on-shell massive fermions with momenta $p_{1}$ (quark) and $p_{2}$ (antiquark). The explicit one-loop self-energies are given by

$$
\begin{array}{r}
-\imath \Sigma\left(\not p_{1}\right)=\imath g_{\mathrm{S}}^{2} C_{\mathrm{F}} \int_{\ell}\left(\prod_{i=1,3} G_{\mathrm{F}}\left(q_{i}\right)\right) \gamma^{\mu}\left(-\not q_{3}+M\right) \gamma^{\nu} d_{\mu \nu}\left(q_{1}\right), \\
-\imath \Sigma\left(-\not p_{2}\right)=\imath g_{\mathrm{S}}^{2} C_{\mathrm{F}} \int_{\ell}\left(\prod_{i=1,2} G_{\mathrm{F}}\left(q_{i}\right)\right) \gamma^{\mu}\left(-q_{2}+M\right) \gamma^{\nu} d_{\mu \nu}\left(q_{1}\right) .
\end{array}
$$

In these expressions, we keep the same internal momenta $q_{i}$ that were used to define the vertex corrections in section 3 (see also figure 8 in appendix $\mathrm{C}$ ). This will allow us to reuse the same momentum mappings already defined to treat the vertex corrections. Because of the symmetry $\not p_{1} \leftrightarrow-\not p_{2}$, the unintegrated expression for the antiquark self-energy corrections can be deduced from those of the quark. Thus, we consider in the next only the quark self-energy. In the Feynman gauge

$$
\Sigma\left(\not p_{1}\right)=g_{\mathrm{S}}^{2} C_{\mathrm{F}} \int_{\ell}\left(\prod_{i=1,3} G_{\mathrm{F}}\left(q_{i}\right)\right)\left((d-2) q_{3}+d M\right) .
$$

Working in the on-shell renormalisation scheme (OS), the renormalised self-energy fulfills

$$
\Sigma_{R}\left(\not p_{1}=M\right)=0,\left.\quad \frac{d \Sigma_{R}\left(\not p_{1}\right)}{d \not p_{1}}\right|_{\not \not_{1}=M}=0,
$$

from where the wave function and mass renormalisation corrections are given by

$$
\Delta Z_{2}=\left.\frac{\partial}{\partial \not p_{1}} \Sigma\left(\not p_{1}\right)\right|_{\not p_{1}=M}, \quad \Delta Z_{M}^{\mathrm{OS}}=-\frac{1}{M} \Sigma\left(\not p_{1}=M\right) .
$$

Explicitly, from eq. (7.4) we obtain

$$
\begin{aligned}
\Delta Z_{2}\left(p_{1}\right) & =-g_{\mathrm{S}}^{2} C_{\mathrm{F}} \int_{\ell} G_{\mathrm{F}}\left(q_{1}\right) G_{\mathrm{F}}\left(q_{3}\right)\left((d-2) \frac{q_{1} \cdot p_{2}}{p_{1} \cdot p_{2}}+4 M^{2}\left(1-\frac{q_{1} \cdot p_{2}}{p_{1} \cdot p_{2}}\right) G_{\mathrm{F}}\left(q_{3}\right)\right), \\
\Delta Z_{M}^{\mathrm{OS}}\left(p_{1}\right) & =-g_{\mathrm{S}}^{2} C_{\mathrm{F}} \int_{\ell} G_{\mathrm{F}}\left(q_{1}\right) G_{\mathrm{F}}\left(q_{3}\right)\left((d-2) \frac{q_{1} \cdot p_{2}}{p_{1} \cdot p_{2}}+2\right) .
\end{aligned}
$$


It is worth to stress that the expression of the wave function renormalisation constant in eq. (7.7) tends smoothly in the massless limit to the corresponding expression given in ref. [39]. It is also relevant to notice that the term proportional to $M^{2}\left(G_{\mathrm{F}}\left(q_{3}\right)\right)^{2}$ leads to soft divergences when $q_{1}$ gets on-shell. They are expected to cancel the soft divergences of the squared amplitudes of the real corrections. The dual representation of the mass renormalisation constant is straightforward from the LTD theorem. The term $M^{2}\left(G_{\mathrm{F}}\left(q_{3}\right)\right)^{2}$ in eq. (7.7), however, introduces double poles that need to be treated specifically [31]. The final dual representations for both renormalisation factors are

$$
\begin{aligned}
\Delta Z_{2}\left(p_{1}\right)= & g_{\mathrm{S}}^{2} C_{\mathrm{F}} \int_{\ell}\left[\frac{\tilde{\delta}\left(q_{1}\right)}{-2 q_{1} \cdot p_{1}}\left((d-2) \frac{q_{1} \cdot p_{2}}{p_{1} \cdot p_{2}}-\frac{4 M^{2}}{2 q_{1} \cdot p_{1}}\left(1-\frac{q_{1} \cdot p_{2}}{p_{1} \cdot p_{2}}\right)\right)+\frac{\tilde{\delta}\left(q_{3}\right)}{2 M^{2}+2 q_{3} \cdot p_{1}}\right. \\
& \left.\times\left((d-2)\left(1+\frac{q_{3} \cdot p_{2}}{p_{1} \cdot p_{2}}\right)+\frac{4 M^{2}}{p_{1} \cdot p_{2}}\left(-\frac{\mathbf{q}_{3} \cdot \mathbf{p}_{2}}{2\left(q_{3,0}^{(+)}\right)^{2}}+\frac{\left(q_{3,0}^{(+)}+p_{1,0}\right) q_{3} \cdot p_{2}}{q_{3,0}^{(+)}\left(2 M^{2}+2 q_{3} \cdot p_{1}\right)}\right)\right)\right], \\
\Delta Z_{M}^{\mathrm{OS}}\left(p_{1}\right)= & g_{\mathrm{S}}^{2} C_{\mathrm{F}} \int_{\ell}\left[\frac{\tilde{\delta}\left(q_{1}\right)}{-2 q_{1} \cdot p_{1}}\left((d-2) \frac{q_{1} \cdot p_{2}}{p_{1} \cdot p_{2}}+2\right)+\frac{\tilde{\delta}\left(q_{3}\right)}{2 M^{2}+2 q_{3} \cdot p_{1}}\left((d-2) \frac{q_{3} \cdot p_{2}}{p_{1} \cdot p_{2}}+d\right)\right],
\end{aligned}
$$

or in term of dual variables defined in section 3

$$
\begin{aligned}
\Delta Z_{2}\left(p_{1}\right)= & g_{\mathrm{S}}^{2} C_{\mathrm{F}}\left[\int \frac { 2 d [ \xi _ { 1 , 0 } ] d [ v _ { 1 } ] } { 1 - \beta ( 1 - 2 v _ { 1 } ) } \left(-(d-2) \frac{\xi_{1,0}\left(1+\beta\left(1-2 v_{1}\right)\right)}{1+\beta^{2}}\right.\right. \\
& \left.+\frac{2 m^{2}}{1-\beta\left(1-2 v_{1}\right)}\left(\frac{1}{\xi_{1,0}}-\frac{1+\beta\left(1-2 v_{1}\right)}{1+\beta^{2}}\right)\right) \\
& +\int \frac{2 \xi_{3}^{2} d\left[\xi_{3}\right] d\left[v_{3}\right]}{\xi_{3,0}\left(\xi_{3,0}-\beta \xi_{3}\left(1-2 v_{3}\right)+m^{2}\right)}\left((d-2)\left(1+\frac{\xi_{3,0}+\beta \xi_{3}\left(1-2 v_{3}\right)}{1+\beta^{2}}\right)\right. \\
& \left.\left.+\frac{2 m^{2}}{\left(1+\beta^{2}\right) \xi_{3,0}}\left(\frac{\beta \xi_{3}\left(1-2 v_{3}\right)}{\xi_{3,0}}+\frac{\left(1+\xi_{3,0}\right)\left(\xi_{3,0}+\beta \xi_{3}\left(1-2 v_{3}\right)\right)}{\xi_{3,0}-\beta \xi_{3}\left(1-2 v_{3}\right)+m^{2}}\right)\right)\right] \\
\Delta Z_{M}^{\mathrm{OS}}\left(p_{1}\right)= & g_{\mathrm{S}}^{2} C_{\mathrm{F}}\left[-\int \frac{2 d\left[\xi_{1,0}\right] d\left[v_{1}\right]}{1-\beta\left(1-2 v_{1}\right)}\left((d-2) \frac{\xi_{1,0}\left(1+\beta\left(1-2 v_{1}\right)\right)}{1+\beta^{2}}+2\right)\right. \\
& \left.+\int \frac{2 \xi_{3}^{2} d\left[\xi_{3}\right] d\left[v_{3}\right]}{\xi_{3,0}\left(\xi_{3,0}-\beta \xi_{3}\left(1-2 v_{3}\right)+m^{2}\right)}\left((d-2) \frac{\xi_{3,0}+\beta \xi_{3}\left(1-2 v_{3}\right)}{1+\beta^{2}}+d\right)\right] .
\end{aligned}
$$

\section{UV renormalisation}

We shall now remove the UV divergences of the renormalisation constants by defining suitable integrand level UV counter-terms. These UV counter-terms are obtained by expanding eqs. (7.7) and (7.8) around the UV propagator $G_{\mathrm{F}}\left(q_{\mathrm{UV}}\right)=1 /\left(q_{\mathrm{UV}}^{2}-\mu_{\mathrm{UV}}^{2}+i 0\right)$, where $q_{\mathrm{UV}}=\ell+k_{\mathrm{UV}}$ with $k_{\mathrm{UV}}$ arbitrary [36, 39, 45]. The simplest choice is $k_{\mathrm{UV}}=0$. We 
obtain

$$
\begin{aligned}
\Delta Z_{2}^{\mathrm{UV}}\left(p_{1}\right)= & -(d-2) g_{\mathrm{S}}^{2} C_{\mathrm{F}} \int_{\ell}\left(G_{\mathrm{F}}\left(q_{\mathrm{UV}}\right)\right)^{2}\left(1+\frac{q_{\mathrm{UV}} \cdot p_{2}}{p_{1} \cdot p_{2}}\right) \\
& \times\left(1-G_{\mathrm{F}}\left(q_{\mathrm{UV}}\right)\left(2 q_{\mathrm{UV}} \cdot p_{1}+\mu_{\mathrm{UV}}^{2}\right)\right) \\
\Delta Z_{M}^{\mathrm{OS}, \mathrm{UV}}\left(p_{1}\right)= & -g_{\mathrm{S}}^{2} C_{\mathrm{F}} \int_{\ell}\left(G_{\mathrm{F}}\left(q_{\mathrm{UV}}\right)\right)^{2}\left(d+(d-2) \frac{q_{\mathrm{UV}} \cdot p_{2}}{p_{1} \cdot p_{2}}\right) \\
& \times\left(1-G_{\mathrm{F}}\left(q_{\mathrm{UV}}\right)\left(2 q_{\mathrm{UV}} \cdot p_{1}+2 d^{-1} \mu_{\mathrm{UV}}^{2}\right)\right)
\end{aligned}
$$

whose integrated form is

$$
\begin{aligned}
\Delta Z_{2}^{\mathrm{UV}} & =-\widetilde{S}_{\epsilon} \frac{\alpha_{\mathrm{S}}}{4 \pi} C_{\mathrm{F}}\left(\frac{\mu_{\mathrm{UV}}^{2}}{\mu^{2}}\right)^{-\epsilon} \frac{1-\epsilon^{2}}{\epsilon}, \\
\Delta Z_{M}^{\mathrm{OS}, \mathrm{UV}} & =-\widetilde{S}_{\epsilon} \frac{\alpha_{\mathrm{S}}}{4 \pi} C_{\mathrm{F}}\left(\frac{\mu_{\mathrm{UV}}^{2}}{\mu^{2}}\right)^{-\epsilon} \frac{3}{\epsilon}
\end{aligned}
$$

The sub-leading terms in eq. (8.1), which are proportional to $\mu_{\mathrm{UV}}^{2}$, have been adjusted in such a way that only the UV poles in eq. (7.1) are subtracted at $\mathcal{O}\left(\epsilon^{0}\right)$. Therefore

$$
\Delta Z_{2}^{\mathrm{IR}}=\Delta Z_{2}-\Delta Z_{2}^{\mathrm{UV}}, \quad \Delta Z_{M}^{\mathrm{OS}, \mathrm{IR}}=\Delta Z_{M}^{\mathrm{OS}}-\Delta Z_{M}^{\mathrm{OS}, \mathrm{UV}},
$$

only contain IR singularities, including the finite terms which are scheme dependent.

The dual representation of eq. (8.1) requires to evaluate the residue of poles of second and third order $[31,36,39]$ located at $q_{\mathrm{UV}, 0}^{(+)}=\sqrt{\mathbf{q}_{\mathrm{UV}}^{2}+\mu_{\mathrm{UV}}^{2}-\imath 0}$. We obtain

$$
\begin{aligned}
\Delta Z_{2}^{\mathrm{UV}}= & -(d-2) g_{\mathrm{S}}^{2} C_{\mathrm{F}} \int_{\ell} \frac{\tilde{\delta}\left(q_{\mathrm{UV}}\right)}{2\left(q_{\mathrm{UV}, 0}^{(+)}\right)^{2}}\left[\left(1-\frac{\mathbf{q}_{\mathrm{UV}} \cdot \mathbf{p}_{2}}{p_{1} \cdot p_{2}}\right)\right. \\
& \left.\times\left(1-\frac{3\left(2 \mathbf{q}_{\mathrm{UV}} \cdot \mathbf{p}_{1}-\mu_{\mathrm{UV}}^{2}\right)}{4\left(q_{\mathrm{UV}, 0}^{(+)}\right)^{2}}\right)-\frac{p_{1,0} p_{2,0}}{2 p_{1} \cdot p_{2}}\right] \\
\Delta Z_{M}^{\mathrm{OS}, \mathrm{UV}}= & -g_{\mathrm{S}}^{2} C_{\mathrm{F}} \int_{\ell} \frac{\tilde{\delta}\left(q_{\mathrm{UV}}\right)}{2\left(q_{\mathrm{UV}, 0}^{(+)}\right)^{2}}\left[\left(d-(d-2) \frac{\mathbf{q}_{\mathrm{UV}} \cdot \mathbf{p}_{2}}{p_{1} \cdot p_{2}}\right)\right. \\
& \left.\times\left(1-\frac{3\left(2 \mathbf{q}_{\mathrm{UV}} \cdot \mathbf{p}_{1}-2 d^{-1} \mu_{\mathrm{UV}}^{2}\right)}{4\left(q_{\mathrm{UV}, 0}^{(+)}\right)^{2}}\right)-(d-2) \frac{p_{1,0} p_{2,0}}{2 p_{1} \cdot p_{2}}\right]
\end{aligned}
$$

Then, we use the parametrisation

$$
\begin{aligned}
q_{\mathrm{UV}}^{\mu} & =\frac{\sqrt{s_{12}}}{2}\left(\xi_{\mathrm{UV}, 0}, 2 \xi_{\mathrm{UV}} \sqrt{v_{\mathrm{UV}}\left(1-v_{\mathrm{UV}}\right)} \mathbf{e}_{\mathrm{UV}, \perp}, \xi_{\mathrm{UV}}\left(1-2 v_{\mathrm{UV}}\right)\right), \\
\xi_{\mathrm{UV}, 0} & =\sqrt{m_{\mathrm{UV}}^{2}+\xi_{\mathrm{UV}}^{2}}
\end{aligned}
$$


with $m_{\mathrm{UV}}=2 \mu_{\mathrm{UV}} / \sqrt{s_{12}}$, and the UV counter-term get the form

$$
\begin{aligned}
\Delta Z_{2}^{\mathrm{UV}}= & -(d-2) g_{\mathrm{S}}^{2} C_{\mathrm{F}} \int d\left[\xi_{\mathrm{UV}}\right] d\left[v_{\mathrm{UV}}\right] \frac{2 \xi_{\mathrm{UV}}^{2}}{\xi_{\mathrm{UV}, 0}^{3}}\left[\left(1+\frac{\beta \xi_{\mathrm{UV}}\left(1-2 v_{\mathrm{UV}}\right)}{2\left(1+\beta^{2}\right)}\right)\right. \\
& \left.\times\left(1-\frac{3\left(2 \beta \xi_{\mathrm{UV}}\left(1-2 v_{\mathrm{UV}}\right)-m_{\mathrm{UV}}^{2}\right)}{4 \xi_{\mathrm{UV}, 0}^{2}}\right)-\frac{1}{2\left(1+\beta^{2}\right)}\right], \\
\Delta Z_{M}^{\mathrm{OS}, \mathrm{UV}}= & -g_{\mathrm{S}}^{2} C_{\mathrm{F}} \int d\left[\xi_{\mathrm{UV}}\right] d\left[v_{\mathrm{UV}}\right] \frac{2 \xi_{\mathrm{UV}}^{2}}{\xi_{\mathrm{UV}, 0}^{3}}\left[\left(d+(d-2) \frac{\beta \xi_{\mathrm{UV}}\left(1-2 v_{\mathrm{UV}}\right)}{2\left(1+\beta^{2}\right)}\right)\right. \\
& \left.\times\left(1-\frac{3\left(2 \beta \xi_{\mathrm{UV}}\left(1-2 v_{\mathrm{UV}}\right)-2 d^{-1} m_{\mathrm{UV}}^{2}\right)}{4 \xi_{\mathrm{UV}, 0}^{2}}\right)-\frac{d-2}{2\left(1+\beta^{2}\right)}\right] .
\end{aligned}
$$

Similarly, we should subtract the UV singularities of the $q \bar{q} A$ interaction vertex, with $A=\{\phi, \gamma, Z\}$ for the explicit examples that we will consider later. As for the self-energy contributions, the UV counter-term is obtained by expanding the vertex corrections around the UV propagator $G_{\mathrm{F}}\left(q_{\mathrm{UV}}\right)=1 /\left(q_{\mathrm{UV}}^{2}-\mu_{\mathrm{UV}}^{2}+i 0\right)$. In the Feynman gauge, the generic expression of the vertex UV counter-term reads

$$
\boldsymbol{\Gamma}_{A, \mathrm{UV}}^{(1)}=g_{\mathrm{S}}^{2} C_{\mathrm{F}} \int_{\ell}\left(G_{\mathrm{F}}\left(q_{\mathrm{UV}}\right)\right)^{3}\left[\gamma^{\nu} q_{\mathrm{UV}} \boldsymbol{\Gamma}_{A}^{(0)} q_{\mathrm{UV}} \gamma_{\nu}-d_{A, \mathrm{UV}} \mu_{\mathrm{UV}}^{2} \boldsymbol{\Gamma}_{A}^{(0)}\right]
$$

where the tree-level vertices $\boldsymbol{\Gamma}_{A}^{(0)}$ are given in eq. (C.1) of appendix C. The $\mu_{\mathrm{UV}}^{2}$ term is sub-leading and the coefficient $d_{\mathrm{UV}}$ is adjusted to subtract only the UV pole. Performing the explicit calculation, we find that in the $\overline{\mathrm{MS}}$ scheme these coefficients are

$$
d_{\phi, \mathrm{UV}}=d+4, \quad d_{\gamma, \mathrm{UV}}=d_{Z, \mathrm{UV}}=d .
$$

Notice that this choice of the sub-leading contributions differs from $d_{\gamma, \mathrm{UV}}=4$ proposed in ref. [45]. The difference is, however, of $\mathcal{O}(\epsilon)$. The integration of the vertex UV counter-term leads to the result

$$
\boldsymbol{\Gamma}_{A, \mathrm{UV}}^{(1)}=\widetilde{S}_{\epsilon} \frac{\alpha_{\mathrm{S}}}{4 \pi} C_{\mathrm{F}} \boldsymbol{\Gamma}_{A}^{(0)}\left(\frac{\mu_{\mathrm{UV}}^{2}}{\mu^{2}}\right)^{-\epsilon} \frac{c_{A, \mathrm{UV}}}{\epsilon}
$$

with

$$
c_{\phi, \mathrm{UV}}=4, \quad c_{\gamma, \mathrm{UV}}=c_{Z, \mathrm{UV}}=1
$$

that translates into

$$
\left\langle\mathcal{M}_{A}^{(0)} \mid \mathcal{M}_{A, \mathrm{UV}}^{(1)}\right\rangle=\widetilde{S}_{\epsilon} \frac{\alpha_{\mathrm{S}}}{4 \pi} C_{\mathrm{F}}\left|\mathcal{M}_{A}^{(0)}\right|^{2}\left(\frac{\mu_{\mathrm{UV}}^{2}}{\mu^{2}}\right)^{-\epsilon} \frac{c_{A, \mathrm{UV}}}{\epsilon}
$$

The dual representation of eq. (8.7) is (see ref. [39])

$$
\begin{aligned}
\boldsymbol{\Gamma}_{A, \mathrm{UV}}^{(1)}= & g_{\mathrm{S}}^{2} C_{\mathrm{F}} \int_{\ell} \frac{\tilde{\delta}\left(q_{\mathrm{UV}}\right)}{8\left(q_{\mathrm{UV}, 0}^{(+)}\right)^{2}}\left[\gamma^{\nu} \gamma^{0} \boldsymbol{\Gamma}_{A}^{(0)} \gamma^{0} \gamma_{\nu}\right. \\
& \left.-\frac{3}{\left(q_{\mathrm{UV}, 0}^{(+)}\right)^{2}}\left[\gamma^{\nu}(\gamma \cdot \mathbf{q u V}) \boldsymbol{\Gamma}_{A}^{(0)}(\gamma \cdot \mathbf{q u V}) \gamma_{\nu}-d_{A, \mathrm{UV}} \mu_{\mathrm{UV}}^{2} \boldsymbol{\Gamma}_{A}^{(0)}\right]\right] .
\end{aligned}
$$


After an explicit calculation, we obtain for the vertex UV counter-terms

$$
\begin{aligned}
\left\langle\mathcal{M}_{\phi}^{(0)} \mid \mathcal{M}_{\phi, \mathrm{UV}}^{(1)}\right\rangle= & g_{\mathrm{S}}^{2} C_{\mathrm{F}}\left|\mathcal{M}_{\phi}^{(0)}\right|^{2} \int d\left[\xi_{\mathrm{UV}}\right] d\left[v_{\mathrm{UV}}\right] \frac{2 \xi_{\mathrm{UV}}^{2}}{\xi_{\mathrm{UV}, 0}^{3}}\left(7-2 \epsilon-\frac{3 \xi_{\mathrm{UV}}^{2}}{\xi_{\mathrm{UV}, 0}^{2}}\right) \\
\left\langle\mathcal{M}_{\gamma}^{(0)} \mid \mathcal{M}_{\gamma, \mathrm{UV}}^{(1)}\right\rangle= & g_{\mathrm{S}}^{2} C_{\mathrm{F}} \int d\left[\xi_{\mathrm{UV}}\right] d\left[v_{\mathrm{UV}}\right]\left[\frac{2\left(e e_{q}\right)^{2} C_{A}}{1-\epsilon} f\left(\xi_{\mathrm{UV}}, v_{\mathrm{UV}}\right)\right. \\
& \left.+\left|\mathcal{M}_{\gamma}^{(0)}\right|^{2} \frac{\xi_{\mathrm{UV}}^{2}}{\xi_{\mathrm{UV}, 0}^{3}}\left(7-4 \epsilon-\frac{3 \xi_{\mathrm{UV}}^{2}}{\xi_{\mathrm{UV}, 0}^{2}}\left(1+4 v_{\mathrm{UV}}\left(1-v_{\mathrm{UV}}\right)\right)\right)\right], \\
\left\langle\mathcal{M}_{Z}^{(0)} \mid \mathcal{M}_{Z, \mathrm{UV}}^{(1)}\right\rangle= & g_{\mathrm{S}}^{2} C_{\mathrm{F}} \int d\left[\xi_{\mathrm{UV}}\right] d\left[v_{\mathrm{UV}}\right]\left[\frac{2 g_{V, q}^{2} C_{A}}{1-\epsilon} f\left(\xi_{\mathrm{UV}}, v_{\mathrm{UV}}\right)\right. \\
& \left.+\left|\mathcal{M}_{Z}^{(0)}\right|^{2} \frac{\xi_{\mathrm{UV}}^{2}}{\xi_{\mathrm{UV}, 0}^{3}}\left(7-4 \epsilon-\frac{3 \xi_{\mathrm{UV}}^{2}}{\xi_{\mathrm{UV}, 0}^{2}}\left(1+4 v_{\mathrm{UV}}\left(1-v_{\mathrm{UV}}\right)\right)\right)\right],
\end{aligned}
$$

where the function

$$
f\left(\xi_{\mathrm{UV}}, v_{\mathrm{UV}}\right)=24 M^{2} \frac{\xi_{\mathrm{UV}}^{4}}{\xi_{\mathrm{UV}, 0}^{5}}\left(\epsilon\left(1-2 v_{\mathrm{UV}}\right)^{2}+6 v_{\mathrm{UV}}\left(1-v_{\mathrm{UV}}\right)-1\right)
$$

integrates to zero and does not contribute to the renormalisation of the vertex. However, this additional term is necessary to achieve a local cancellation of the UV behaviour.

The UV divergences of the wave function cancel exactly the UV divergences of the vertex corrections for photons and $Z$ bosons, because conserved currents or partially conserved currents, as the vector and axial ones, do not get renormalised. The corresponding dual representations, however, do not cancel each other at integrand level. In particular, the wave function renormalisation contains linear UV singularities that cancel upon integration. Also, the vertex UV counter-term contains terms that are proportional to the mass and cancel upon integration. The contribution of all this spurious terms is, however, crucial to cancel locally all the UV singularities. The coupling to scalar particles, on the contrary, needs to be renormalised

$$
Y_{q}^{0} \mu_{0}^{\epsilon}=Y_{q} \mu^{\epsilon}\left(1-\frac{\alpha_{\mathrm{S}}}{4 \pi} \frac{3 C_{\mathrm{F}}}{\epsilon}\right)+\mathcal{O}\left(\alpha_{\mathrm{S}}^{2}\right)
$$

\section{LTD four-dimensional unsubtraction for physical processes}

We have already defined all the necessary ingredients to test the four dimensional implementation of NLO corrections to physical processes in the LTD framework. In particular, we will compute the NLO QCD corrections to the decay rate $A^{*} \rightarrow q \bar{q}(g)$, with $A=\phi, \gamma, Z$. The actual implementation is indeed independent of the decaying particle. The renormalised one-loop amplitude is given by

$$
\left|\mathcal{M}_{A}^{(1, \mathrm{R})}\right\rangle=\left|\mathcal{M}_{A}^{(1)}\right\rangle-\left|\mathcal{M}_{A}^{(1, \mathrm{UV})}\right\rangle+\frac{1}{2}\left(\Delta Z_{2}^{\mathrm{IR}}\left(p_{1}\right)+\Delta Z_{2}^{\mathrm{IR}}\left(p_{2}\right)\right)\left|\mathcal{M}_{A}^{(0)}\right\rangle
$$


where $\left|\mathcal{M}_{A}^{(1, \mathrm{UV})}\right\rangle$ is the unintegrated UV counter-term of the one-loop vertex correction, $\left|\mathcal{M}_{A}^{(1)}\right\rangle$, and $Z_{2}^{\mathrm{IR}}\left(p_{i}\right)$ are the IR components of the quark and antiquark self-energy corrections. From the renormalised one-loop amplitude $\left|\mathcal{M}_{A}^{(1, \mathrm{R})}\right\rangle$, which contains only IR singularities, we construct the LTD representation of the renormalised virtual decay rate

$$
\Gamma_{\mathrm{V}, A}^{(1, \mathrm{R})}=\frac{1}{2 \sqrt{s_{12}}} \sum_{i=1}^{3} \int d \Phi_{1 \rightarrow 2} 2 \operatorname{Re}\left\langle\mathcal{M}_{A}^{(0)} \mid \mathcal{M}_{A}^{(1, \mathrm{R})}\left(\tilde{\delta}\left(q_{i}\right)\right)\right\rangle
$$

The corresponding dual amplitudes for the vertex corrections are given explicitly in eqs. (C.7), (C.8) and (C.9). As for the toy scalar example presented in section 6, the real contributions are implemented by splitting the real phase-space in two domains

$$
\widetilde{\Gamma}_{\mathrm{R}, A, i}^{(1)}=\frac{1}{2 \sqrt{s_{12}}} \int d \Phi_{1 \rightarrow 3}\left|\mathcal{M}_{A \rightarrow q \bar{q} g}^{(0)}\right|^{2} \mathcal{R}_{i}\left(y_{i r}^{\prime}<y_{j r}^{\prime}\right), \quad i, j=\{1,2\}
$$

with $\Gamma_{\mathrm{R}, A}^{(1)}=\widetilde{\Gamma}_{\mathrm{R}, A, 1}^{(1)}+\widetilde{\Gamma}_{\mathrm{R}, A, 2}^{(1)}$ the real total decay rate. The real emission squared amplitudes are given in eq. (C.5). In each of the real-phase space domain we introduce one of the momentum mappings defined in section 5. The sum of the virtual and real corrections in eq. (9.2) and eq. (9.3) is a single integral in the loop three-momentum. It is UV and IR finite locally and thus can be calculated numerically with $\epsilon=0$. We follow the same numerical implementation as for the scalar example presented in section 6 , and compare the numerical output with the analytical total decay rate, which has the form

$$
\Gamma_{A}^{(1)}=\frac{\alpha_{\mathrm{S}}}{4 \pi} C_{\mathrm{F}}\left[\Gamma_{A}^{(0)}\left(F\left(x_{S}\right)+2\left(c_{A, \mathrm{UV}}-1\right) \log \left(\frac{\mu_{\mathrm{UV}}^{2}}{s_{12}}\right)\right)+G_{A}\left(x_{S}\right)\right]+\mathcal{O}(\epsilon) .
$$

Our results, normalised to the LO decay rate $\Gamma_{A}^{(0)}$, are presented in figure 6 for a scalar and pseudoscalar, and in figure 7 for vector bosons. The computing time is similar to that of the toy scalar example presented in section 6; a few minutes to generate all the points. The agreement with the analytic prediction is excellent in all the cases. Moreover, the massless limit, i.e. $x_{S} \rightarrow 0$, is also well-defined and we recover the known results. This is a very subtle point, because individual contributions in DREG are not smoothly well-defined in that limit.

\section{Conclusions and outlook}

In this article, we have generalised the four-dimensional unsubtraction method [36-40] to deal with massive particles. Based in the LTD theorem, it exploits the possibility of expressing virtual amplitudes as phase-space integrals, and the fact that threshold and IR singularities are always restricted to a compact region of the loop three-momentum integration domain. This is a crucial point, because it allows to establish a momentum mapping to generate the real-emission on-shell kinematics starting from the Born level momenta and the loop three-momentum, in such a way that a local cancellation of both IR and UV singularities can be achieved without introducing IR subtractions. In particular 

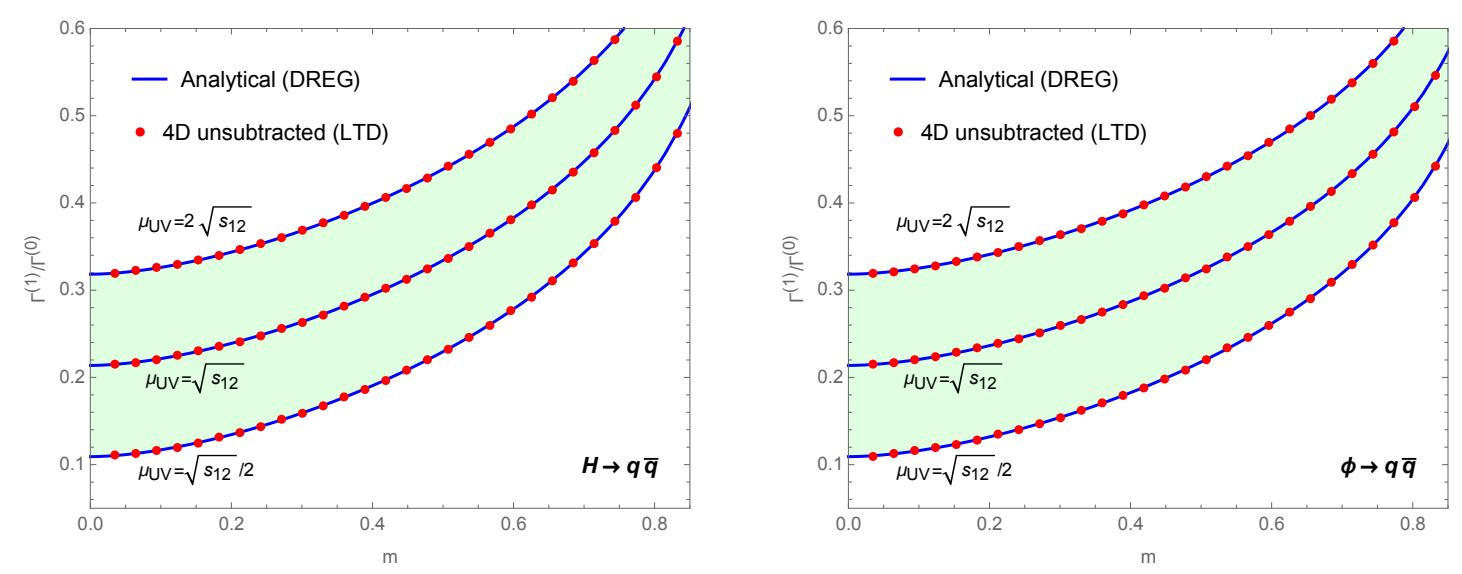

Figure 6. Total decay rate at NLO for scalar and pseudoscalar particles into a pair of heavy quarks as a function of the mass, normalised to the LO. In the left panel, we consider a standard Higgs boson, whilst in the right panel we plot the decay rate for a pseudoscalar particle $\left(c_{q}=\right.$ $1 / 2$ ). The solid blue lines correspond to the usual DREG analytic result, while the red dots were computed numerically within the LTD unsubtracted method. We also consider a renormalisation scale variation, in the range $1 / 2<\mu_{\mathrm{UV}} / \sqrt{s_{12}}<2$.

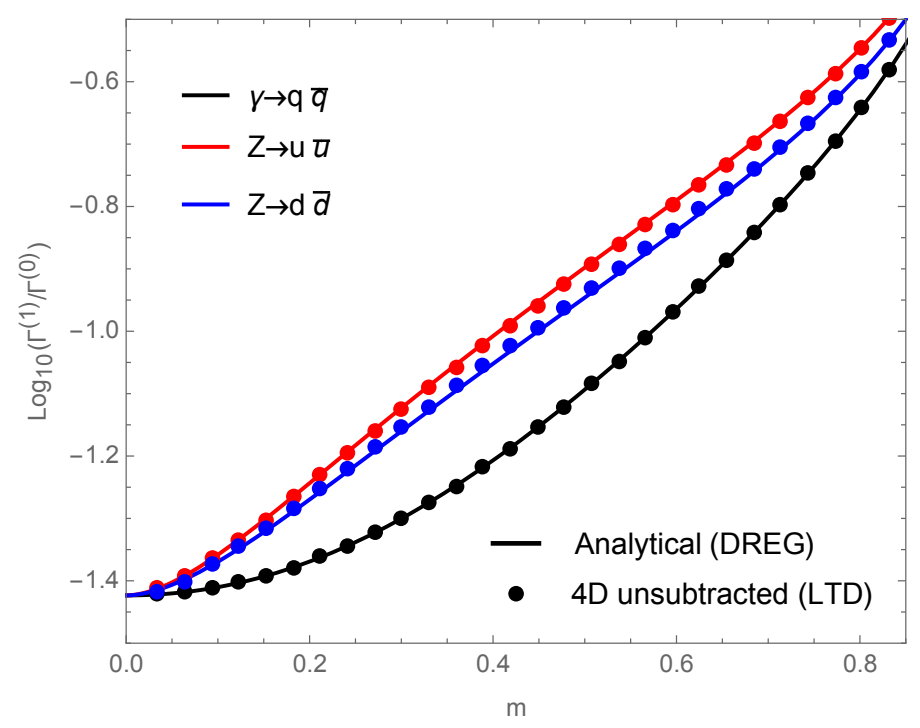

Figure 7. Total decay rate at NLO for off-shell vector particles into a pair of heavy quarks as a function of the mass, normalised to the LO. We consider three physical cases: $\gamma^{*} \rightarrow q \bar{q}$ (black), $Z^{*} \rightarrow u \bar{u}$ (red, up-type quarks) and $Z^{*} \rightarrow d \bar{d}$ (blue, down-type quarks). Solid lines corresponds to the results within the DREG approach, whilst the dots we obtained with the LTD unsubtracted method. 
for the massive case, we have defined a general momentum mapping that accounts properly for the quasi-collinear configurations.

First, we started by inquiring in the computation of the scalar three-point function with massive particles within the LTD approach. Besides recovering the previously known results, the analysis of the integration domains of the dual contributions allowed us to understand the origin of its singular structure. Then, we illustrated the local cancellation of IR and quasi-collinear configurations with a toy scalar example.

The full cancellation of IR singularities requires the contributions of the self-energy corrections. Thus, we defined unintegrated versions of the quark wave function and mass renormalisation factors in the on-shell renormalisation (OS) scheme. Compared to the massless case, this is a non-trivial case because the OS scheme is built at integral level and DREG leads to very simple results after integration. In any case, the unintegrated renormalisation constants are completely general and lead to a fully local cancellation of the remaining IR singularities. The treatment of UV singularities was also discussed carefully, and we constructed suitable unintegrated UV counter-terms for both self-energy and vertex corrections, which reproduce successfully the $\overline{\mathrm{MS}}$ conditions and also lead to a fully local cancellation of UV singularities.

Finally, we tested the LTD four-dimensional unsubtraction to compute NLO QCD corrections to the decay rate of scalar and vector particles into a pair of massive quarks. The results were compared with the standard DREG expressions, and we found an impressive agreement. In particular, the transition to the massless limit is smooth because the quasi-collinear configurations of the real and virtual corrections are matched at integrand level. With the results presented in this paper, LTD four-dimensional unsubtraction can be applied to any multipartonic process involving heavy quarks, and other heavy particles.

\section{Acknowledgments}

This work has been supported by CONICET Argentina, by the Spanish Government and ERDF funds from the European Commission (Grants No. FPA2014-53631-C2-1-P and SEV-2014-0398) and by Generalitat Valenciana under Grant No. PROMETEOII/2013/007. FDM acknowledges support from Generalitat Valenciana (GRISOLIA/2015/035).

\section{A Phase-space}

The phase-space for a $1 \rightarrow 2$ decay with final-state particles of equal masses, $p_{i}^{2}=M^{2}$ with $i=1,2$ and $s_{12}$ the virtuality of the decaying particle, is given by

$$
\int d \Phi_{1 \rightarrow 2}=\frac{\Gamma(1-\epsilon) \beta^{1-2 \epsilon}}{2(4 \pi)^{1-\epsilon} \Gamma(2-2 \epsilon)}\left(\frac{s_{12}}{\mu^{2}}\right)^{-\epsilon},
$$

with $\beta=\sqrt{1-m^{2}}$ and $m^{2}=4 M^{2} / s_{12}$. The corresponding $1 \rightarrow 3$ phase-space of the real radiation correction with an additional massless particle in the final state, $\left(p_{r}^{\prime}\right)^{2}=0$, is given by

$$
d \Phi_{1 \rightarrow 3}=\frac{(4 \pi)^{\epsilon-2} s_{12}}{\Gamma(1-\epsilon)}\left(\frac{s_{12}}{\mu^{2}}\right)^{-\epsilon}\left(\int d \Phi_{1 \rightarrow 2}\right) \beta^{-1+2 \epsilon} \theta\left(h_{p}\right) h_{p}^{-\epsilon} d y_{1 r}^{\prime} d y_{2 r}^{\prime},
$$


with

$$
h_{p}=\left(1-y_{1 r}^{\prime}-y_{2 r}^{\prime}\right) y_{1 r}^{\prime} y_{2 r}^{\prime}-\frac{m^{2}}{4}\left(y_{1 r}^{\prime}+y_{2 r}^{\prime}\right)^{2},
$$

where $y_{i r}^{\prime}=2 p_{i}^{\prime} \cdot p_{r}^{\prime} / s_{12}$. In order to integrate analytically the real radiation contribution, it is convenient to use the change of variables suggested in ref. [54], i.e.

$$
y_{1 r}^{\prime}=g(z) w, \quad y_{2 r}^{\prime}=g(z) z w, \quad g(z)=\frac{\left(z-x_{S}\right)\left(1-x_{S} z\right)}{z(1+z)\left(1+x_{S}\right)^{2}},
$$

which allows to factorise the function $h_{p}$ in eq. (A.3) according to

$$
h_{p}=g(z)^{3} z(1+z) w^{2}(1-w) .
$$

In consequence, the phase-space limits, which are determined by the quadratic function $h_{p}$, simplify to $z \in\left[x_{S}, x_{S}^{-1}\right]$ and $w \in[0,1]$. The first integral in $w$ can easily be obtained by keeping the exact $\epsilon$-dependence. The second integral in $z$, however, requires to expand the result up to $\mathcal{O}\left(\epsilon^{0}\right)$ before integration.

\section{B Unification of coordinates}

In order to avoid local mismatches in the $\epsilon$-expansion close to the singular regions, it is necessary to unify the coordinate system and express all the on-shell momenta $q_{i}$ in terms of a single loop three-momentum [36]. This is a crucial point to obtain integrable expressions directly at integrand level in four space-time dimensions. Since $q_{3}=\ell$, we parametrise the on-shell momenta $q_{1}$ and $q_{2}$ by using the variables $(\xi, v) \equiv\left(\xi_{3}, v_{3}\right)$. Notice that each $q_{i}$ is set on-shell inside the corresponding dual contribution. Hence, by using the associated dispersion relations, we work with the spatial components of the momenta and fix the energy to fulfill the on-shell condition. From eq. (3.8), the spatial components of the loop momenta must satisfy

$$
\begin{aligned}
\mathbf{q}_{1} & =\frac{\sqrt{s_{12}}}{2} \xi_{1,0}\left(2 \sqrt{v_{1}\left(1-v_{1}\right)} \mathbf{e}_{1, \perp}, 1-2 v_{1}\right) \\
& =\mathbf{q}_{3}+\mathbf{p}_{1}=\frac{\sqrt{s_{12}}}{2}\left(2 \xi_{3} \sqrt{v_{3}\left(1-v_{3}\right)} \mathbf{e}_{3, \perp}, \xi_{3}\left(1-2 v_{3}\right)+\beta\right),
\end{aligned}
$$

which leads to a system of equations, whose solution is

$$
\begin{aligned}
\xi_{1,0} & =\sqrt{(\beta+\xi)^{2}-4 \beta v \xi} \\
v_{1} & =\frac{1}{2}\left(1-\frac{\beta+(1-2 v) \xi}{\xi_{1,0}}\right) .
\end{aligned}
$$

Similarly, we can express $\left(\xi_{2}, v_{2}\right)$ in terms of $(\xi, v)$ from $\mathbf{q}_{2}=\mathbf{q}_{3}$. This leads to the trivial replacement $\left(\xi_{2}, v_{2}\right) \rightarrow(\xi, v)$. It is worth appreciating that this change of reference frame is well defined because the argument of the square root in eq. (B.2) is always positive. In fact,

$$
(\xi+\beta)^{2}-4 \beta \xi v>(\xi+\beta)^{2}-4 \xi \beta=(\xi-\beta)^{2}>0
$$



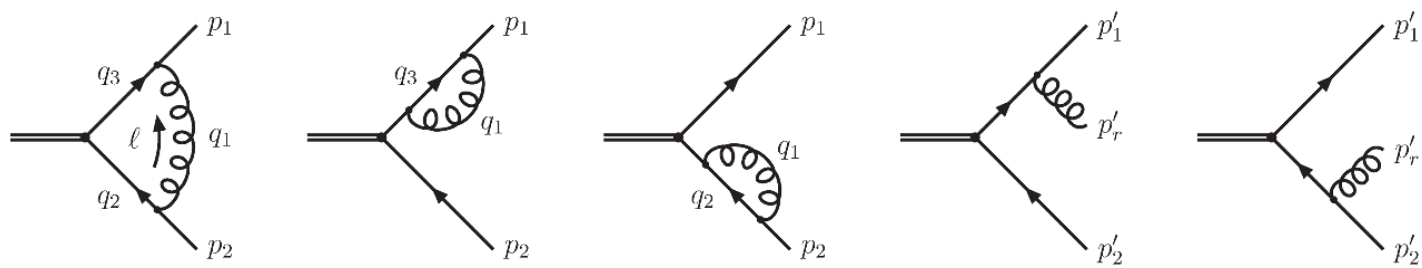

Figure 8. Momentum configuration of the NLO QCD corrections to the process $A^{*} \rightarrow q \bar{q}(g)$, under the assumption that the decaying particle does not couple to gluons.

due to $\beta<1$. Then, the associated Jacobian is given by

$$
\mathcal{J}(\xi, v)=\frac{\xi^{2}}{(\xi+\beta)^{2}-4 v \beta \xi},
$$

whose massless limit (i.e. $\beta \rightarrow 1$ ) agrees with the expressions found in refs. [36, 39].

\section{LTD amplitudes for $A^{*} \rightarrow q \bar{q}(g)$}

In this appendix, we collect the dual amplitudes and real squared amplitudes contributing to the NLO QCD corrections to the processes $A^{*} \rightarrow q \bar{q}(g)$, with $A=\{\phi, \gamma, Z\}$. The tree-level vertices are given by

$$
\begin{aligned}
& \Gamma_{\phi}^{(0)}=\imath Y_{q}\left(1+c_{q} \gamma^{5}\right) \\
& \boldsymbol{\Gamma}_{\gamma}^{(0)}=\imath e e_{q} \gamma^{\mu}, \\
& \boldsymbol{\Gamma}_{Z}^{(0)}=\imath \gamma^{\mu}\left(g_{V, q}+g_{A, q} \gamma^{5}\right) .
\end{aligned}
$$

The corresponding Born squared amplitudes, averaged over the initial-state polarisations, are

$$
\begin{aligned}
\left|\mathcal{M}_{\phi \rightarrow q q \bar{q}}^{(0)}\right|^{2} & =2 s_{12} Y_{q}^{2} C_{A}\left(\beta^{2}+c_{q}^{2}\right) \\
\left|\mathcal{M}_{\gamma \rightarrow q \bar{q}}^{(0)}\right|^{2} & =2 s_{12}\left(e e_{q}\right)^{2} C_{A}\left(1+\frac{m^{2}}{2(1-\epsilon)}\right) \\
\left|\mathcal{M}_{Z \rightarrow q \bar{q}}^{(0)}\right|^{2} & =2 s_{12} C_{A} \frac{2(1-\epsilon)}{3-2 \epsilon}\left(g_{V, q}^{2}\left(1+\frac{m^{2}}{2(1-\epsilon)}\right)+g_{A, q}^{2} \beta^{2}\right) .
\end{aligned}
$$

The momentum configuration of the NLO QCD corrections is represented in figure 8 .

The squared amplitudes for the real process $A^{*} \rightarrow q\left(p_{1}^{\prime}\right)+\bar{q}\left(p_{2}^{\prime}\right)+g\left(p_{r}^{\prime}\right)$, are given by

$$
\begin{aligned}
\left|\mathcal{M}_{\phi \rightarrow q \bar{q} g}^{(0)}\right|^{2}= & 4 g_{\mathrm{S}}^{2} C_{\mathrm{F}}\left[s_{12}^{-1}\left|\mathcal{M}_{\phi \rightarrow q \bar{q}}^{(0)}\right|^{2} \frac{h_{p}}{2\left(y_{1 r}^{\prime} y_{2 r}^{\prime}\right)^{2}}\right. \\
& \left.+Y_{q}^{2}\left(1+c_{q}^{2}\right) C_{A}(1-\epsilon)\left(1+\frac{y_{2 r}^{\prime}}{y_{1 r}^{\prime}}\right)\right]+(1 \leftrightarrow 2), \\
\left|\mathcal{M}_{\gamma \rightarrow q \bar{q} g}^{(0)}\right|^{2}= & 4 g_{\mathrm{S}}^{2} C_{\mathrm{F}}\left[s_{12}^{-1}\left|\mathcal{M}_{\gamma \rightarrow q \bar{q}}^{(0)}\right|^{2} \frac{h_{p}}{2\left(y_{1 r}^{\prime} y_{2 r}^{\prime}\right)^{2}}+\left(e e_{q}\right)^{2} C_{A}\left((1-\epsilon) \frac{y_{2 r}^{\prime}}{y_{1 r}^{\prime}}-\epsilon\right)\right]+(1 \leftrightarrow 2),
\end{aligned}
$$




$$
\begin{aligned}
\left|\mathcal{M}_{Z \rightarrow q \bar{q} g}^{(0)}\right|^{2}= & 4 g_{\mathrm{S}}^{2} C_{\mathrm{F}}\left[s_{12}^{-1}\left|\mathcal{M}_{Z \rightarrow q \bar{q}}^{(0)}\right|^{2} \frac{h_{p}}{2\left(y_{1 r}^{\prime} y_{2 r}^{\prime}\right)^{2}}+C_{A} \frac{2(1-\epsilon)}{3-2 \epsilon}\right. \\
& \left.\times\left(\left(g_{V, q}^{2}+g_{A, q}^{2}\right)\left((1-\epsilon) \frac{y_{2 r}^{\prime}}{y_{1 r}^{\prime}}-\epsilon\right)+g_{A, q}^{2} \frac{m^{2}}{2}\left(1+\frac{y_{2 r}^{\prime}}{y_{1 r}^{\prime}}\right)\right)\right]+(1 \leftrightarrow 2),
\end{aligned}
$$

where the function $h_{p}$ is defined in eq. (A.3).

The vertex corrections to the process $A^{*} \rightarrow q \bar{q}$ are given by the dual amplitudes

$$
\begin{aligned}
& \left\langle\mathcal{M}_{A}^{(0)} \mid \mathcal{M}_{A}^{(1)}\left(\tilde{\delta}\left(q_{1}\right)\right)\right\rangle=g_{\mathrm{S}}^{2} C_{\mathrm{F}} \int_{\ell} \tilde{\delta}\left(q_{1}\right)\left[-\frac{\left|\mathcal{M}_{A}^{(0)}\right|^{2} s_{12}\left(1+\beta^{2}\right)}{\left(2 q_{1} \cdot p_{1}\right)\left(2 q_{1} \cdot p_{2}\right)}+\mathcal{G}_{A}\left(\tilde{\delta}\left(q_{1}\right)\right)\right], \\
& \left\langle\mathcal{M}_{A}^{(0)} \mid \mathcal{M}_{A}^{(1)}\left(\tilde{\delta}\left(q_{2}\right)\right)\right\rangle=g_{\mathrm{S}}^{2} C_{\mathrm{F}} \int_{\ell} \tilde{\delta}\left(q_{2}\right)\left[\frac{\left|\mathcal{M}_{A}^{(0)}\right|^{2} 4 q_{2} \cdot p_{1}}{\left(2 M^{2}-2 q_{2} \cdot p_{2}\right)\left(s_{12}-2 q_{2} \cdot p_{12}+\imath 0\right)}+\mathcal{G}_{A}\left(\tilde{\delta}\left(q_{2}\right)\right)\right], \\
& \left\langle\mathcal{M}_{A}^{(0)} \mid \mathcal{M}_{A}^{(1)}\left(\tilde{\delta}\left(q_{3}\right)\right)\right\rangle=g_{\mathrm{S}}^{2} C_{\mathrm{F}} \int_{\ell} \tilde{\delta}\left(q_{3}\right)\left[-\frac{\left|\mathcal{M}_{A}^{(0)}\right|^{2} 4 q_{3} \cdot p_{2}}{\left(2 M^{2}+2 q_{3} \cdot p_{1}\right)\left(s_{12}+2 q_{3} \cdot p_{12}\right)}+\mathcal{G}_{A}\left(\tilde{\delta}\left(q_{3}\right)\right)\right],
\end{aligned}
$$

where $\left|\mathcal{M}_{A}^{(0)}\right|^{2}$ are the Born squared amplitudes in eq. (C.4), and the process dependent functions $\mathcal{G}_{A}\left(\tilde{\delta}\left(q_{i}\right)\right)$ for the process $\phi^{*} \rightarrow q \bar{q}$ are

$$
\begin{aligned}
& \mathcal{G}_{\phi}\left(\tilde{\delta}\left(q_{1}\right)\right)=2 Y_{q}^{2} C_{A}\left(1+\beta^{2}+2 c_{q}^{2}\left(\frac{s_{12}}{2 q_{1} \cdot p_{1}}-\frac{s_{12}}{2 q_{1} \cdot p_{2}}\right)\right), \\
& \mathcal{G}_{\phi}\left(\tilde{\delta}\left(q_{2}\right)\right)=2 Y_{q}^{2} C_{A}\left(-\frac{m^{2} s_{12}}{2 M^{2}-2 q_{2} \cdot p_{2}}+\frac{2\left(1-(2-\epsilon) \beta^{2}\right) s_{12}}{s_{12}-2 q_{2} \cdot p_{12}+\imath 0}-c_{q}^{2} \frac{2(1-\epsilon) s_{12}}{s_{12}-2 q_{2} \cdot p_{12}+\imath 0}\right), \\
& \mathcal{G}_{\phi}\left(\tilde{\delta}\left(q_{3}\right)\right)=2 Y_{q}^{2} C_{A}\left(-\frac{m^{2} s_{12}}{2 M^{2}+2 q_{3} \cdot p_{1}}+\frac{2\left(1-(2-\epsilon) \beta^{2}\right) s_{12}}{s_{12}+2 q_{3} \cdot p_{12}}-c_{q}^{2} \frac{2(1-\epsilon) s_{12}}{s_{12}+2 q_{3} \cdot p_{12}}\right) .
\end{aligned}
$$

For $\gamma^{*} \rightarrow q \bar{q}$, they are

$$
\begin{aligned}
& \mathcal{G}_{\gamma}\left(\tilde{\delta}\left(q_{1}\right)\right)=2\left(e e_{q}\right)^{2} C_{A}\left(\left(2+\frac{m^{2}}{2(1-\epsilon)}\right)\left(\frac{s_{12}}{2 q_{1} \cdot p_{1}}-\frac{s_{12}}{2 q_{1} \cdot p_{2}}\right)+2\right), \\
& \mathcal{G}_{\gamma}\left(\tilde{\delta}\left(q_{2}\right)\right)=2\left(e e_{q}\right)^{2} C_{A}\left(\frac{m^{2} s_{12}}{2(1-\epsilon)\left(2 M^{2}-2 q_{2} \cdot p_{2}\right)}+\frac{2 \epsilon s_{12}+4 q_{2} \cdot p_{1}}{s_{12}-2 q_{2} \cdot p_{12}+\imath 0}\right), \\
& \mathcal{G}_{\gamma}\left(\tilde{\delta}\left(q_{3}\right)\right)=2\left(e e_{q}\right)^{2} C_{A}\left(\frac{m^{2} s_{12}}{2(1-\epsilon)\left(2 M^{2}+2 q_{3} \cdot p_{1}\right)}+\frac{2 \epsilon s_{12}-4 q_{3} \cdot p_{2}}{s_{12}+2 q_{3} \cdot p_{12}}\right) .
\end{aligned}
$$

Finally, for $Z^{*} \rightarrow q \bar{q}$,

$$
\begin{aligned}
\mathcal{G}_{Z}\left(\tilde{\delta}\left(q_{1}\right)\right)= & 2 C_{A} \frac{2(1-\epsilon)}{3-2 \epsilon}\left[g_{V, q}^{2}\left(\left(2+\frac{m^{2}}{2(1-\epsilon)}\right)\left(\frac{s_{12}}{2 q_{1} \cdot p_{1}}-\frac{s_{12}}{2 q_{1} \cdot p_{2}}\right)+2\right)\right. \\
& \left.+g_{A, q}^{2}\left(\left(1+\beta^{2}\right)\left(\frac{s_{12}}{2 q_{1} \cdot p_{1}}-\frac{s_{12}}{2 q_{1} \cdot p_{2}}+1\right)-\frac{m^{2}}{2}\left(\frac{q_{1} \cdot p_{2}}{q_{1} \cdot p_{1}}-\frac{q_{1} \cdot p_{1}}{q_{1} \cdot p_{2}}\right)\right)\right], \\
\mathcal{G}_{Z}\left(\tilde{\delta}\left(q_{2}\right)\right)= & 2 C_{A} \frac{2(1-\epsilon)}{3-2 \epsilon}\left[g_{V, q}^{2}\left(\frac{m^{2} s_{12}}{2(1-\epsilon)\left(2 M^{2}-2 q_{2} \cdot p_{2}\right)}+\frac{2 \epsilon s_{12}+4 q_{2} \cdot p_{1}}{s_{12}-2 q_{2} \cdot p_{12}+\imath 0}\right)\right. \\
& \left.+g_{A, q}^{2}\left(-\frac{m^{2}\left(2 q_{2} \cdot p_{12}+s_{12}\right)}{2\left(2 M^{2}-2 q_{2} \cdot p_{2}\right)}+\frac{\left(m^{2}+2 \epsilon \beta^{2}\right) s_{12}+4 q_{2} \cdot p_{1}}{s_{12}-2 q_{2} \cdot p_{12}+\imath 0}\right)\right],
\end{aligned}
$$




$$
\begin{aligned}
\mathcal{G}_{Z}\left(\tilde{\delta}\left(q_{3}\right)\right)= & 2 C_{A} \frac{2(1-\epsilon)}{3-2 \epsilon}\left[g_{V, q}^{2}\left(\frac{m^{2} s_{12}}{2(1-\epsilon)\left(2 M^{2}+2 q_{3} \cdot p_{1}\right)}+\frac{2 \epsilon s_{12}-4 q_{3} \cdot p_{2}}{s_{12}+2 q_{3} \cdot p_{12}}\right)\right. \\
& \left.+g_{A, q}^{2}\left(-\frac{m^{2}\left(2 q_{3} \cdot p_{12}+s_{12}\right)}{2\left(2 M^{2}+2 q_{3} \cdot p_{1}\right)}+\frac{\left(m^{2}+2 \epsilon \beta^{2}\right) s_{12}-4 q_{3} \cdot p_{2}}{s_{12}+2 q_{3} \cdot p_{12}}\right)\right] .
\end{aligned}
$$

Open Access. This article is distributed under the terms of the Creative Commons Attribution License (CC-BY 4.0), which permits any use, distribution and reproduction in any medium, provided the original author(s) and source are credited.

\section{References}

[1] J.C. Collins, Renormalization: An Introduction to Renormalization, The Renormalization Group, and the Operator Product Expansion, Cambridge Monographs on Mathematical Physics, volume 26, Cambridge University Press, Cambridge U.K. (1986) [ISBN: 9780521311779, 9780511867392].

[2] T. Kinoshita, Mass singularities of Feynman amplitudes, J. Math. Phys. 3 (1962) 650 [INSPIRE].

[3] T.D. Lee and M. Nauenberg, Degenerate Systems and Mass Singularities, Phys. Rev. 133 (1964) B1549 [INSPIRE].

[4] C.G. Bollini and J.J. Giambiagi, Dimensional Renormalization: The Number of Dimensions as a Regularizing Parameter, Nuovo Cim. B 12 (1972) 20 [INSPIRE].

[5] G. 't Hooft and M.J.G. Veltman, Regularization and Renormalization of Gauge Fields, Nucl. Phys. B 44 (1972) 189 [INSPIRE].

[6] G.M. Cicuta and E. Montaldi, Analytic renormalization via continuous space dimension, Lett. Nuovo Cim. 4 (1972) 329 [INSPIRE].

[7] J.F. Ashmore, A Method of Gauge Invariant Regularization, Lett. Nuovo Cim. 4 (1972) 289 [INSPIRE].

[8] Z. Kunszt and D.E. Soper, Calculation of jet cross-sections in hadron collisions at order $\alpha_{s}^{3}$, Phys. Rev. D 46 (1992) 192 [InSPIRE].

[9] S. Frixione, Z. Kunszt and A. Signer, Three jet cross-sections to next-to-leading order, Nucl. Phys. B 467 (1996) 399 [hep-ph/9512328] [INSPIRE].

[10] S. Catani and M.H. Seymour, The Dipole formalism for the calculation of QCD jet cross-sections at next-to-leading order, Phys. Lett. B 378 (1996) 287 [hep-ph/9602277] [INSPIRE].

[11] S. Catani and M.H. Seymour, A General algorithm for calculating jet cross-sections in NLO QCD, Nucl. Phys. B 485 (1997) 291 [Erratum ibid. B 510 (1998) 503] [hep-ph/9605323] [INSPIRE].

[12] A. Gehrmann-De Ridder, T. Gehrmann and E.W.N. Glover, Antenna subtraction at NNLO, JHEP 09 (2005) 056 [hep-ph/0505111] [INSPIRE].

[13] S. Seth and S. Weinzierl, Numerical integration of subtraction terms, Phys. Rev. D 93 (2016) 114031 [arXiv: 1605.06646] [INSPIRE]. 
[14] S. Catani and M. Grazzini, An NNLO subtraction formalism in hadron collisions and its application to Higgs boson production at the LHC, Phys. Rev. Lett. 98 (2007) 222002 [hep-ph/0703012] [INSPIRE].

[15] S. Catani, L. Cieri, G. Ferrera, D. de Florian and M. Grazzini, Vector boson production at hadron colliders: a fully exclusive QCD calculation at NNLO, Phys. Rev. Lett. 103 (2009) 082001 [arXiv: 0903.2120] [INSPIRE].

[16] M. Czakon, A novel subtraction scheme for double-real radiation at NNLO, Phys. Lett. B 693 (2010) 259 [arXiv: 1005.0274] [INSPIRE].

[17] P. Bolzoni, G. Somogyi and Z. Trócsányi, A subtraction scheme for computing QCD jet cross sections at NNLO: integrating the iterated singly-unresolved subtraction terms, JHEP 01 (2011) 059 [arXiv: 1011.1909] [INSPIRE].

[18] V. Del Duca, C. Duhr, G. Somogyi, F. Tramontano and Z. Trócsányi, Higgs boson decay into b-quarks at NNLO accuracy, JHEP 04 (2015) 036 [arXiv:1501.07226] [INSPIRE].

[19] R. Boughezal, C. Focke, X. Liu and F. Petriello, $W$-boson production in association with a jet at next-to-next-to-leading order in perturbative QCD, Phys. Rev. Lett. 115 (2015) 062002 [arXiv: 1504.02131] [INSPIRE].

[20] J. Gaunt, M. Stahlhofen, F.J. Tackmann and J.R. Walsh, N-jettiness Subtractions for NNLO QCD Calculations, JHEP 09 (2015) 058 [arXiv: 1505.04794] [InSPIRE].

[21] V. Del Duca et al., Jet production in the CoLoRFulNNLO method: event shapes in electron-positron collisions, Phys. Rev. D 94 (2016) 074019 [arXiv:1606.03453] [INSPIRE].

[22] V. Del Duca, C. Duhr, A. Kardos, G. Somogyi and Z. Trócsányi, Three-jet production in electron-positron collisions using the CoLoRFulNNLO method, Phys. Rev. Lett. 117 (2016) 152004 [arXiv: 1603.08927] [INSPIRE].

[23] S. Catani, S. Dittmaier, M.H. Seymour and Z. Trócsányi, The Dipole formalism for next-to-leading order QCD calculations with massive partons, Nucl. Phys. B 627 (2002) 189 [hep-ph/0201036] [INSPIRE].

[24] A. Gehrmann-De Ridder and M. Ritzmann, NLO Antenna Subtraction with Massive Fermions, JHEP 07 (2009) 041 [arXiv: 0904.3297] [InSPIRE].

[25] G. Abelof, O. Dekkers and A. Gehrmann-De Ridder, Antenna subtraction with massive fermions at NNLO: Double real initial-final configurations, JHEP 12 (2012) 107 [arXiv: 1210.5059] [INSPIRE].

[26] G. Abelof, A. Gehrmann-De Ridder, P. Maierhofer and S. Pozzorini, NNLO QCD subtraction for top-antitop production in the $q \bar{q}$ channel, JHEP 08 (2014) 035 [arXiv:1404.6493] [INSPIRE].

[27] R. Bonciani, S. Catani, M. Grazzini, H. Sargsyan and A. Torre, The $q_{T}$ subtraction method for top quark production at hadron colliders, Eur. Phys. J. C 75 (2015) 581 [arXiv: 1508.03585] [INSPIRE].

[28] S. Catani, T. Gleisberg, F. Krauss, G. Rodrigo and J.-C. Winter, From loops to trees by-passing Feynman's theorem, JHEP 09 (2008) 065 [arXiv:0804.3170] [INSPIRE].

[29] G. Rodrigo, S. Catani, T. Gleisberg, F. Krauss and J.-C. Winter, From multileg loops to trees (by-passing Feynman's Tree Theorem), Nucl. Phys. Proc. Suppl. 183 (2008) 262 [arXiv: 0807.0531] [INSPIRE]. 
[30] I. Bierenbaum, S. Catani, P. Draggiotis and G. Rodrigo, A Tree-Loop Duality Relation at Two Loops and Beyond, JHEP 10 (2010) 073 [arXiv: 1007.0194] [INSPIRE].

[31] I. Bierenbaum, S. Buchta, P. Draggiotis, I. Malamos and G. Rodrigo, Tree-Loop Duality Relation beyond simple poles, JHEP 03 (2013) 025 [arXiv:1211.5048] [INSPIRE].

[32] S. Buchta, G. Chachamis, P. Draggiotis, I. Malamos and G. Rodrigo, On the singular behaviour of scattering amplitudes in quantum field theory, JHEP 11 (2014) 014 [arXiv: 1405.7850] [INSPIRE].

[33] S. Buchta, Theoretical foundations and applications of the Loop-Tree Duality in Quantum Field Theories, arXiv:1509.07167 [INSPIRE].

[34] S. Buchta, G. Chachamis, P. Draggiotis and G. Rodrigo, Numerical implementation of the Loop-Tree Duality method, arXiv:1510.00187 [INSPIRE].

[35] S. Buchta, G. Chachamis, P. Draggiotis, I. Malamos and G. Rodrigo, Towards a Numerical Implementation of the Loop-Tree Duality Method, Nucl. Part. Phys. Proc. 258-259 (2015) 33 [arXiv: 1509.07386] [INSPIRE].

[36] R.J. Hernández-Pinto, G.F.R. Sborlini and G. Rodrigo, Towards gauge theories in four dimensions, JHEP 02 (2016) 044 [arXiv:1506.04617] [INSPIRE].

[37] G.F.R. Sborlini, R.J. Hernández-Pinto and G. Rodrigo, From dimensional regularization to NLO computations in four dimensions, PoS (EPS-HEP2015) 479 [arXiv:1510.01079] [INSPIRE].

[38] G.F.R. Sborlini, Loop-tree duality and quantum field theory in four dimensions, PoS (RADCOR2015) 082 [arXiv: 1601.04634] [INSPIRE].

[39] G.F.R. Sborlini, F. Driencourt-Mangin, R.J. Hernández-Pinto and G. Rodrigo, Four-dimensional unsubtraction from the loop-tree duality, JHEP 08 (2016) 160 [arXiv: 1604.06699] [INSPIRE].

[40] G. Rodrigo, F. Driencourt-Mangin, G.F.R. Sborlini and R.J. Hernández-Pinto, Applications of the loop-tree duality, PoS (LL2016) 037 [arXiv: 1608.01800] [INSPIRE].

[41] D.E. Soper, QCD calculations by numerical integration, Phys. Rev. Lett. 81 (1998) 2638 [hep-ph/9804454] [INSPIRE].

[42] D.E. Soper, Techniques for QCD calculations by numerical integration, Phys. Rev. D 62 (2000) 014009 [hep-ph/9910292] [INSPIRE].

[43] D.E. Soper, Choosing integration points for QCD calculations by numerical integration, Phys. Rev. D 64 (2001) 034018 [hep-ph/0103262] [INSPIRE].

[44] M. Krämer and D.E. Soper, Next-to-leading order numerical calculations in Coulomb gauge, Phys. Rev. D 66 (2002) 054017 [hep-ph/0204113] [INSPIRE].

[45] S. Becker, C. Reuschle and S. Weinzierl, Numerical NLO QCD calculations, JHEP 12 (2010) 013 [arXiv: 1010.4187] [INSPIRE].

[46] S. Becker, C. Reuschle and S. Weinzierl, Efficiency Improvements for the Numerical Computation of NLO Corrections, JHEP 07 (2012) 090 [arXiv: 1205.2096] [INSPIRE].

[47] R. Pittau, A four-dimensional approach to quantum field theories, JHEP 11 (2012) 151 [arXiv: 1208.5457] [INSPIRE].

[48] A.M. Donati and R. Pittau, Gauge invariance at work in FDR: $H \rightarrow \gamma \gamma$, JHEP 04 (2013) 167 [arXiv: 1302.5668] [INSPIRE]. 
[49] R.A. Fazio, P. Mastrolia, E. Mirabella and W.J. Torres Bobadilla, On the Four-Dimensional Formulation of Dimensionally Regulated Amplitudes, Eur. Phys. J. C 74 (2014) 3197 [arXiv: 1404.4783] [INSPIRE].

[50] R.P. Feynman, Quantum theory of gravitation, Acta Phys. Polon. 24 (1963) 697 [InSPIRE].

[51] R.P. Feynman, Closed Loop And Tree Diagrams, in Selected Papers of Richard Feynman With Commentary, L.M. Brown ed., World Scientific (2000), pp. 867-887 [INSPIRE].

[52] R.K. Ellis and G. Zanderighi, Scalar one-loop integrals for QCD, JHEP 02 (2008) 002 [arXiv: 0712.1851] [INSPIRE].

[53] G. Rodrigo, M.S. Bilenky and A. Santamaria, Quark mass effects for jet production in $e^{+} e^{-}$ collisions at the next-to-leading order: Results and applications, Nucl. Phys. B 554 (1999) 257 [hep-ph/9905276] [INSPIRE].

[54] M.S. Bilenky, G. Rodrigo and A. Santamaria, Three jet production at LEP and the bottom quark mass, Nucl. Phys. B 439 (1995) 505 [hep-ph/9410258] [INSPIRE]. 